Research article

urn:1sid:zoobank.org:pub:F8ED2AA9-83C1-4CB8-8327-58C501B6C42A

\title{
Deep-sea nematodes (Comesomatidae) from the Southwest Pacific Ocean: five new species and three new species records
}

\author{
Daniel LEDUC \\ Department of Marine Science, University of Otago, P.O. Box 56, Dunedin, New Zealand \\ National Institute of Water and Atmospheric Research (NIWA) Limited, \\ Private Bag 14-901, Kilbirnie, Wellington, New Zealand \\ Email: daniel.leduc@gmail.com \\ urn:1sid:zoobank.org:author:9393949F-3426-4EE2-8BDE-DEFFACE3D9BC
}

\begin{abstract}
The present study describes five new free-living nematode species and provides three new species records of the family Comesomatidae (genera Cervonema Wieser, 1954, Dorylaimopsis Ditlevsen, 1918, Hopperia Vitiello, 1969, and Kenyanema Muthumbi et al., 1997) from the continental margin of New Zealand, Southwest Pacific. Dichotomous identification keys are provided for all known species of Dorylaimopsis and Hopperia. Cervonema shiae Chen \& Vincx, 2000 is recorded for the first time outside the type locality (Beagle Channel, Chile). C. kaikouraensis sp. nov. is characterised by amphideal fovea with 5.5 turns situated at 1.7 head diameter from anterior end, jointed outer labial setae, equal in length to cephalic setae, sperm dimorphism, and 5-6 small pre-cloacal supplements. C. multispira sp. nov. is characterised by amphideal fovea with 8.0-8.5 turns situated at 2.6-4.0 head diameter from anterior end, cephalic setae 2-3 $\mu \mathrm{m}$ long, slightly shorter than outer labial setae, presence of six uninucleated cells in males (potentially pseudocoelomocytes or supplementary excretory cells), 5 small pre-cloacal supplements, and strongly cuticularised, arcuate spicules with capitulum. C. proberti sp. nov. is characterised by amphideal fovea with 5 turns and located at $<1$ head diameter from anterior end, cephalic setae 1.6-2.0 times longer than outer labial setae, and 8 small pre-cloacal supplements. Dorylaimopsis nodderi sp. nov. is characterised by cuticle with lateral differentiation consisting of three longitudinal rows of larger dots in the pharyngeal and caudal regions, two rows of larger dots in middle region of body, and spicules with rounded ventral projection at one third of spicule length from distal end, giving appearance of a joint. Hopperia ancora sp. nov. is characterised by short conical cephalic setae, spicules with hook-like projection at distal end, gubernaculum with bent apophyses, and 11-13 pre-cloacal supplements. H. beaglense Chen \& Vincx, 1998 is recorded from Kaikoura Canyon, the first record of this species outside the type locality (Beagle Channel, Chile). Kenyanema monorchis Muthumbi et al., 1997 is also recorded for the first time outside the type locality (Indian Ocean).
\end{abstract}

Keywords. Cervonema, Dorylaimopsis, Hopperia, Kenyanema, New Zealand.

Leduc D. 2012. Deep-sea nematodes (Comesomatidae) from the Southwest Pacific Ocean: five new species and three new species records. European Journal of Taxonomy 24: 1-42. http://dx.doi.org/10.5852/ejt.2012.24 


\section{Introduction}

Nematode species of the family Comesomatidae Filipjev, 1918 are common on continental margins worldwide (Soetaert \& Heip 1995; Vanhove et al. 1999; Muthumbi et al. 2011). Little is known, however, about the diversity and distribution of deep-sea ( $>200 \mathrm{~m}$ water depth) Comesomatidae in the Southwest Pacific (Miljutin et al. 2010). Two new species of this family were recently described from the continental slope of New Zealand (Leduc et al. 2012a). The present study describes five new species and provides three new species record belonging to the genera Cervonema Wieser, 1954, Dorylaimopsis Ditlevsen, 1918, Hopperia Vitiello, 1969, and Kenyanema Muthumbi et al., 1997 from the same region.

\section{Material and Methods}

\section{Sampling and laboratory methods}

The present study focussed on three major bathymetric features of the New Zealand Exclusive Economic Zone (EEZ): Challenger Plateau, Chatham Rise and Kaikoura Canyon. Challenger Plateau encompasses water depths ranging from $c .400$ to $3000 \mathrm{~m}$ in an area of generally low biological productivity to the northwest of the South Island, New Zealand (Murphy et al. 2001). Chatham Rise is a submarine ridge that extends eastwards from the South Island of New Zealand, over water depths ranging from c. 250 to $3000 \mathrm{~m}$. The rise lies beneath the Subtropical Front (STF), a region associated with heightened primary productivity (BradfordGrieve et al. 1997; Murphy et al. 2001). Kaikoura Canyon lies to the east of the South Island ( $\left.42^{\circ} \mathrm{S}\right)$, and has been described as one of the most productive deep-sea benthic habitats known (De Leo et al. 2010).

Details of the study sites are given in Leduc et al. (2012a, b). Samples were collected along a transect at $178^{\circ} 30^{\prime} \mathrm{E}$ across Chatham Rise (nine sites, $350-3100 \mathrm{~m}$ water depth) in austral spring (SeptemberOctober) 2001 during National Institute of Water and Atmospheric Research (NIWA) cruise TAN0116. Twenty-three additional sites between 240 and 1300 m water depths on Chatham Rise and Challenger Plateau were sampled in austral autumn-early winter (March-April and May-June) 2007 (NIWA cruises TAN0705 and TAN0707, respectively). Five sites (405-1420 m water depth) were sampled along the Kaikoura Canyon axis in May 2010 during NIWA cruise TAN1006.

Sediment samples were collected using an Ocean Instruments MC-800A multicorer (core internal diameter $=9.5 \mathrm{~cm}$ ). Each sample consisted of one subcore of internal diameter $26 \mathrm{~mm}$ taken to a depth of $5 \mathrm{~cm}$. Samples were fixed in $10 \%$ formalin and stained with Rose Bengal. Samples were subsequently rinsed on a $1 \mathrm{~mm}$ sieve to remove large particles and on a $45 \mu \mathrm{m}$ sieve to retain nematodes. Nematodes were extracted from the remaining sediments by Ludox flotation and transferred to pure glycerol (Somerfield \& Warwick 1996). Species descriptions were made from glycerol mounts using differential interference contrast microscopy and drawings were made with the aid of a camera lucida. All measurements are in $\mu \mathrm{m}$, and all curved structures are measured along the arc. Type specimens are held in the NIWA Invertebrate Collection (NIC), Wellington. Anterior sense organs are defined as follows: papillae ( $<1 \mu \mathrm{m}$ long), setiform papillae $(1-2 \mu \mathrm{m})$, and setae $(>2 \mu \mathrm{m})$ (Leduc \& Wharton 2008).

\begin{tabular}{lll}
\multicolumn{2}{l}{ Abbreviations } \\
$\mathrm{a}$ & $=$ & body length/maximum body diameter \\
$\mathrm{abd}$ & $=$ & anal body diameter \\
$\mathrm{b}$ & $=$ & body length $/$ pharynx length \\
$\mathrm{c}$ & $=$ & body length/tail length \\
$\mathrm{cbd}$ & $=$ & corresponding body diameter \\
$\mathrm{hd}$ & $=$ & head diameter \\
$\mathrm{L}$ & $=$ & body length \\
$\mathrm{n}$ & $=$ & number of specimens \\
$\% \mathrm{~V}$ & $=$ & vulva distance from anterior end of body $\times 100 /$ total body length
\end{tabular}




\title{
Results
}

\author{
Class Chromadorea Inglis, 1983 \\ Order Araeolaimida De Coninck, 1965 \\ Superfamily Axonolaimoidea Filipjev, 1918 \\ Family Comesomatidae Filipjev, 1918 \\ Subfamily Sabatieriinae Filipjev, 1934
}

\section{Diagnosis (modified from Jensen (1979))}

Cephalic sense organs in three separate crowns, with outer labial sensillae and cephalic setae sometimes close together. Buccal cavity weakly cuticularised, anterior portion cup-shaped, sometimes very small; posterior portion narrow, not strongly cuticularised. Spicules bent, usually enlarged proximally. Gubernacular apophyses (when present) usually directed dorsocaudally and paired.

\section{Type genus}

Sabatieria De Rouville, 1903

Genus Cervonema Wieser, 1954

\section{Diagnosis (modified from Chen \& Vincx (2000))}

Sabatieriinae. Body cuticle striated, without punctations; cervical region elongated or short. Six external labial sensillae setiform, usually almost equal in length to four cephalic setae. Amphideal fovea with 4-8 turns. Buccal cavity small, without teeth. Spicules simple, gubernaculum small or absent, minute precloacal supplements present or absent.

\section{Type species}

Cervonema allometricum Wieser, 1954

\section{Remarks}

Interspecific variation in the morphological characters of Cervonema species is relatively limited, and species within this genus should be identified using as many characters as possible (e.g., Barnes et al. 2012). The following characters, however, are particularly useful in species identification: relative length of outer labial setae and cephalic setae, shape and position of amphids, shape and size of spicules, presence/ absence and shape of gubernaculum, and tail length. Other traits, which have not always been included in earlier species descriptions, include presence/absence of jointed outer labial setae and presence /absence of sperm dimorphism. Pre-cloacal supplements are often difficult to observe using light microscopy and should not be used in isolation for defining species.

\section{Cervonema shiae Chen \& Vincx, 2000}

Figs 1-2, Table 1

\section{Material examined}

1 $\delta$ and 2 우, collected 6 Apr. 2007, southern flank of Chatham Rise (1240 m water depth), $44.4864^{\circ} \mathrm{S}$, $177.1407^{\circ} \mathrm{E}$. Characteristics of surface sediment layer $(0-5 \mathrm{~mm})$ : mostly silt/clay $(82.9 \%)$, with very fine sand $(10.2 \%)$, and fine sand $(8.4 \%)$; calcium carbonate content: $38.4 \%$; total organic matter content: 1.9\%; chloroplastic pigment concentration: $3501 \mathrm{ng} / \mathrm{gDW}_{\text {sediment }}$ (NIC 84438). 1 ๙ collected 5 Jun. 2007, Challenger Plateau (532 m water depth), 40.8814'S, $170.8603^{\prime} \mathrm{E}$. Characteristics of surface sediment layer $(0-5 \mathrm{~mm})$ : mostly silt/clay $(67.6 \%)$, with fine sand $(15.2 \%)$, and very fine sand $(11.4 \%)$; calcium carbonate content: $56.0 \%$; total organic matter content: $2.1 \%$; chloroplastic pigment concentration: 1344 $\mathrm{ng} / \mathrm{gDW}_{\text {sediment }}$ (NIC 84439). 


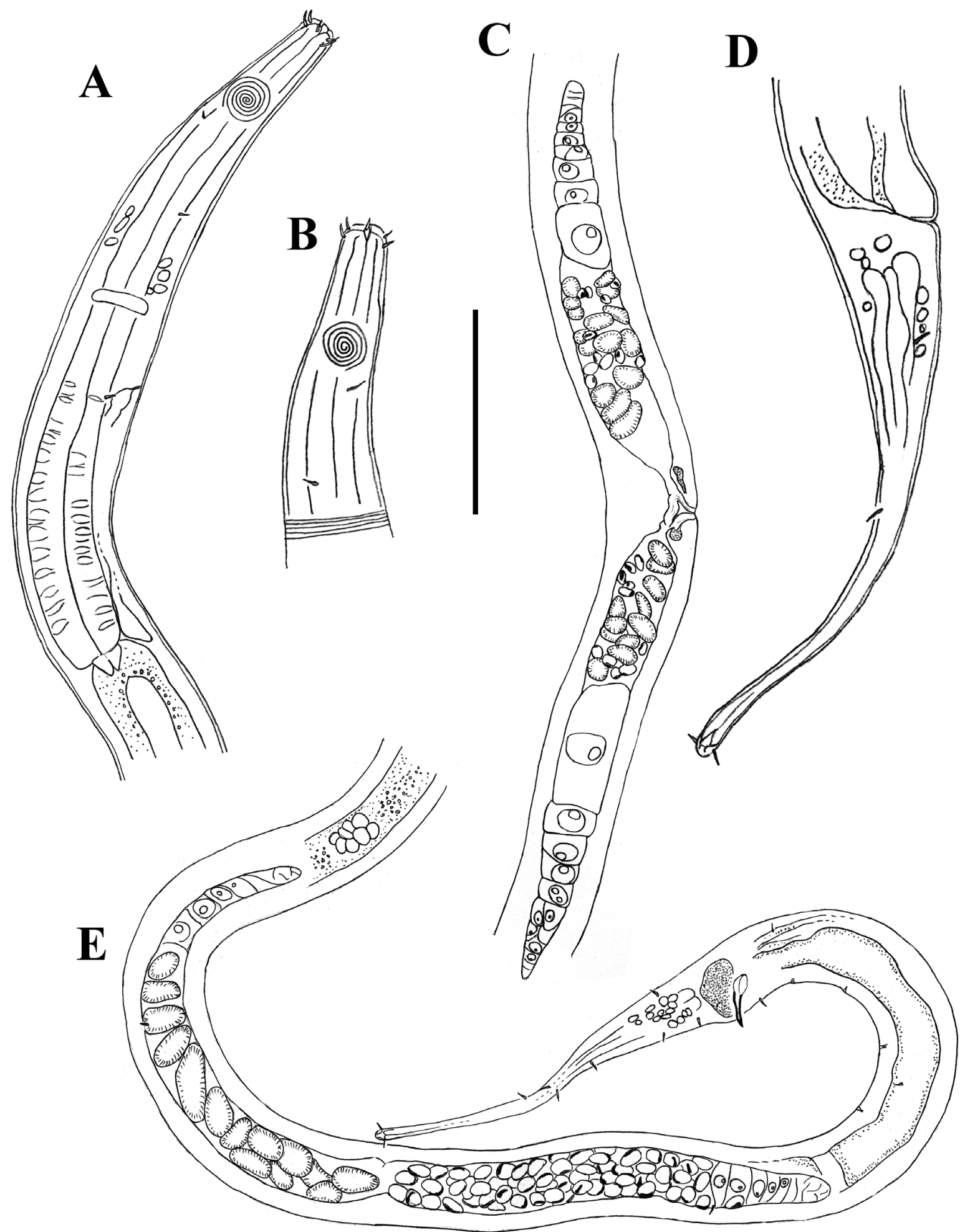

Fig. 1. Cervonema shiae Chen \& Vincx, 2000. A. $\hat{\partial}$ anterior body region. B. Lateral view of $\widehat{\partial}$ head

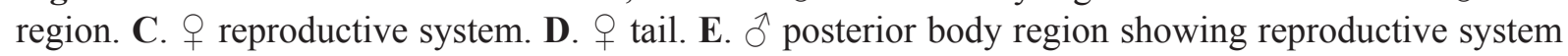
and copulatory apparatus. Scale bar: $\mathrm{A}=40 \mu \mathrm{m} ; \mathrm{B}=35 \mu \mathrm{m} ; \mathrm{C}=65 \mu \mathrm{m} ; \mathrm{D}=30 \mu \mathrm{m} ; \mathrm{E}=60 \mu \mathrm{m}$. 

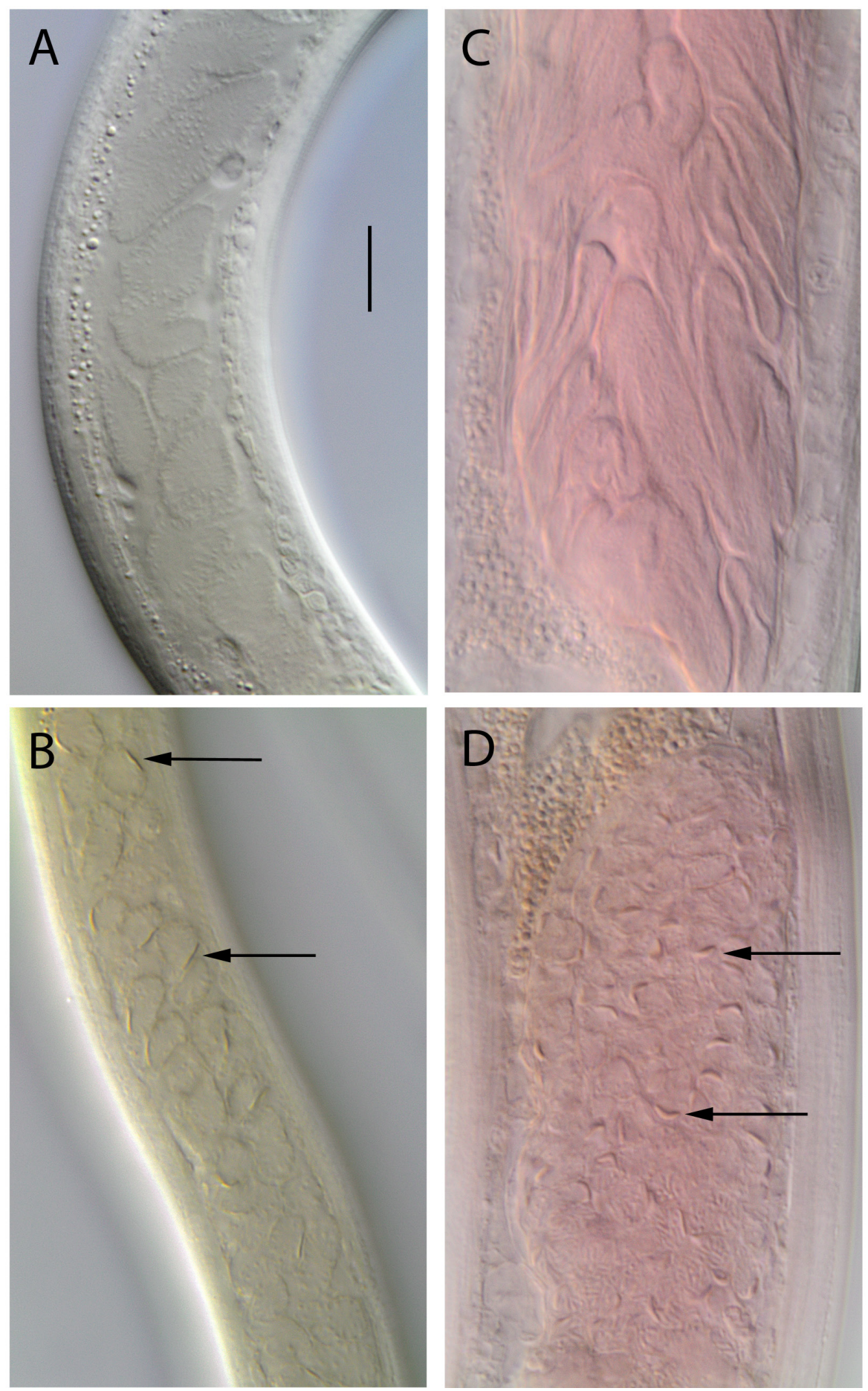

Fig. 2. Cervonema shiae Chen \& Vincx, 2000 and Cervonema kaikouraensis sp. nov. light micrographs. A. Large sperm cells without nuclei in anterior testis of $C$. shiae. B. Small nucleated sperm in posterior testis of C. shiae. C. Large sperm cells without nuclei in anterior testis of C. kaikouraensis sp. nov. D. Small nucleated sperm in posterior testis of C. kaikouraensis sp. nov. Arrows point to the lenticular nuclei situated on the periphery of the small sperm cells in the posterior testes of both species. Scale bar $=10 \mu \mathrm{m}$. 
Table 1. Morphometrics ( $\mu \mathrm{m})$ of Cervonema shiae Chen \& Vincx, 2000 and C. kaikouraensis sp. nov.

\begin{tabular}{|c|c|c|c|c|}
\hline \multirow[t]{2}{*}{ Species } & \multicolumn{2}{|c|}{ Cervonema shiae Chen \& Vincx, 2000} & \multicolumn{2}{|c|}{ Cervonema kaikouraensis sp. nov. } \\
\hline & Males & Females & Male Holotype & Female Paratypes \\
\hline n & 2 & 2 & & 2 \\
\hline $\mathbf{L}$ & $744-827$ & $693-718$ & 1639 & 1876-1959 \\
\hline $\mathbf{a}$ & $30-31$ & $21-24$ & 27 & $18-24$ \\
\hline b & 5 & 5 & 7 & $7-8$ \\
\hline c & $6-7$ & 7 & 8 & $6-7$ \\
\hline Head diam. & $7-8$ & $7-8$ & 11 & $12-13$ \\
\hline Mouth diam. & 2 & 2 & 3 & 3 \\
\hline Length of cephalic setae & $2-3$ & $2-3$ & 6 & 6 \\
\hline Amphid height & 10 & 7 & 13 & $10-11$ \\
\hline Amphid width & 9 & $7-8$ & 12 & $9-12$ \\
\hline Amphid width/cbd (\%) & $75-82$ & $63-73$ & 67 & $41-57$ \\
\hline Amphid from anterior end & $16-20$ & 16 & 19 & $22-23$ \\
\hline Nerve ring from anterior end & $73-79$ & $70-77$ & 117 & 114 \\
\hline Nerve ring cbd & 21 & $19-20$ & 44 & $53-54$ \\
\hline Excretory pore from anterior end & $82-96$ & $81-89$ & 129 & $135-137$ \\
\hline Pharynx length & $150-158$ & $135-143$ & 240 & $255-256$ \\
\hline Pharynx cbd & $23-24$ & $23-26$ & 56 & $69-74$ \\
\hline Pharyngeal bulb diam. & 14 & 16-19 & 37 & $50-55$ \\
\hline Max. body diam. & $25-27$ & $30-33$ & 60 & $83-104$ \\
\hline Spicule length & $11-12$ & - & 32 & - \\
\hline Gubernacular apophyses length & - & - & - & - \\
\hline Anal body diam. & $18-22$ & $18-21$ & 36 & $46-52$ \\
\hline Tail length & $115-122$ & $96-97$ & 198 & $274-302$ \\
\hline Tail length/abd & $5.5-6.4$ & $4.6-5.3$ & 5.5 & $5.8-6.0$ \\
\hline $\mathbf{V}$ & - & $344-389$ & - & $934-955$ \\
\hline$\% \mathrm{~V}$ & - & $50-54$ & - & $49-50$ \\
\hline Vulval body diam. & - & $28-33$ & - & $80-93$ \\
\hline
\end{tabular}

\section{Description}

\section{Males}

Body cylindrical, tapering towards both extremities but more pronounced in tail region. Cuticle faintly striated, no lateral differentiation. Somatic setae short and sparse. Head not set-off by constriction. Inner labial papillae indistinct, six outer labial setae, and four slightly longer cephalic setae, the latter two in separate circles close to each other. Amphideal fovea spiral, at 6.0-6.5 turns, 1.8-2.3 head diameters from anterior. Small buccal cavity, cup-shaped. Pharynx widening posteriorly into a weakly marked elongated bulb. Cardia short. Nerve ring near middle of pharynx length. Secretory-excretory pore near two thirds of pharynx length from anterior. Cellular body of ventral gland small- at level of cardia. Intestine wall consisting mostly of cells containing colourless granules, interspersed with clusters of clear globular inclusions.

Reproductive system diorchic, opposed, outstretched. Anterior testis to left of intestine, posterior testis to right of intestine. Anterior testis with relatively large oval sperm cells $(5-8 \mu \mathrm{m}$ wide by $12-15 \mu \mathrm{m}$ long) without nuclei (Fig. 2A). Posterior testis with smaller, oval-shaped sperm cells (up to $8 \times 5 \mu \mathrm{m}$ ) with 
lenticular nuclei situated peripherally (Fig. 2B). Spicules paired, equal, 0.5-0.6 abd long, with lightly cuticularised, swollen proximal portion, and heavily cuticularised, pointed distal portion. Gubernaculum not observed. Large rectal gland surrounds spicules. Four small, tubular pre-cloacal supplements, one pre-cloacal seta. Tail conico-cylindrical, with several short caudal setae and three short terminal setae. Three caudal glands and spinneret.

\section{Females}

Similar to males, but with slightly larger maximum body diameter, amphideal fovea smaller, 5.0-5.5 turns. Reproductive system didelphic, opposed, ovaries outstretched, with anterior branch to left of intestine and posterior branch to right of intestine. Both types of sperm observed in uterus. Spermathecae not observed. Vulva at median or slightly post median. Granular vaginal glands present, pars proximalis vaginae surrounded by constrictor muscle.

\section{Discussion}

My specimens closely resemble the description of Chen \& Vincx (2000) based on specimens from the Beagle Channel (255-257 m water depth), although specimens from Chatham Rise and Challenger plateau have slightly shorter cephalic setae (0.3-0.4 vs. $0.5 \mathrm{cbd})$ and longer tail (5.5-6.4 vs. 4.8 abd). The specimens are also characterised by sperm dimorphism (anterior testis with large oval sperm cells without nuclei, posterior testis with smaller nucleated sperm cells); Chen \& Vincx (2000), however, did not provide details of the sperm in their description. This is the first record of this species outside the type locality.

\section{Cervonema kaikouraensis sp. nov. urn:1sid:zoobank.org:act:00FCA18B-AB7B-4464-8DBC-A75F17F3BC72}

Figs 2-4, Table 1

\section{Etymology}

This species is named after the type locality.

\section{Material examined}

\section{Holotype}

${ }^{\lambda}$, collected 5 May 2010 , Kaikoura Canyon axis (1061 m water depth), $42.5081^{\circ} \mathrm{S}, 173.6325^{\circ} \mathrm{E}(\mathrm{NIC}$ 84440).

\section{Paratypes}

2 우, same data as holotype (NIC 84441).

\section{Description}

\section{Males}

Body cylindrical, tapering towards both extremities. Cuticle faintly striated, no lateral differentiation. Somatic setae very short and sparse, 1-2 $\mu \mathrm{m}$ long, except in pre-cloacal region, $4 \mu \mathrm{m}$ long. Head not setoff by constriction. Six inner labial papillae, six jointed outer labial setae, and four cephalic setae, not jointed. Amphideal fovea spiral, 5.5 turns, at 1.7 head diameters from anterior. Golden-coloured granular material in lateral, ventral, and dorsal chords, forming two continuous bands (Fig. 4D). Small buccal cavity, cup-shaped. Pharynx gradually widening posteriorly into an elongated bulb. Dorsal pharyngeal gland nucleus conspicuous. Cardia short. Nerve ring near middle of pharynx length. Secretory-excretory pore situated just posteriorly to nerve ring. Cellular body of ventral gland small, at level of cardia. Intestine wall with numerous granules, orange-brown in anterior portion, colourless in posterior portion. 


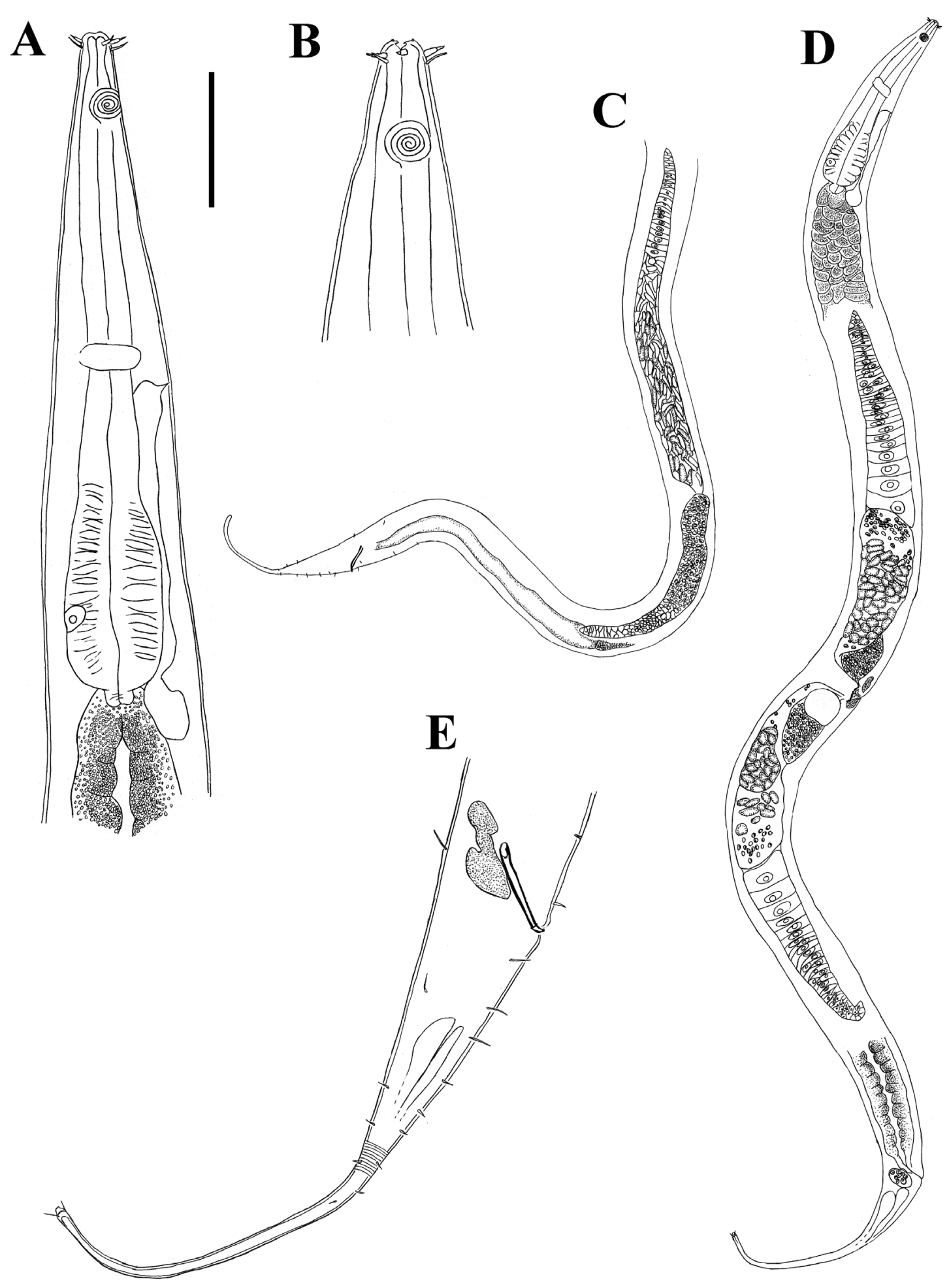

Fig. 3. Cervonema kaikouraensis sp. nov. A. $\hat{\sigma}$ anterior body region. B. $q$ head. C. $\hat{\jmath}$ reproductive system. D. Entire ․ E. $\delta$ copulatory apparatus. Scale bar: $A=50 \mu \mathrm{m} ; \mathrm{B}=30 \mu \mathrm{m} ; \mathrm{C}=170 \mu \mathrm{m}$; $\mathrm{D}=200 \mu \mathrm{m} ; \mathrm{E}=40 \mu \mathrm{m}$. 
Reproductive system diorchic, opposed, outstretched. Anterior testis to left of intestine, posterior testis to right of intestine. Anterior testis with relatively large elongated sperm cells (up to $5 \mu \mathrm{m}$ wide and $20 \mu \mathrm{m}$ long), without nuclei (Fig. 2C). Posterior testis with smaller, globular sperm cells (4-6 $\mu \mathrm{m}$ diameter) with lenticular nuclei situated peripherally (Fig. 2D). Spicules paired, equal, narrow, straight, 0.9 abd long, with small capitulum. Rectal gland present. Five, possibly six tubular pre-cloacal supplements, difficult to observe, one pre-cloacal seta. Tail conico-cylindrical, with several caudal setae, 2-5 $\mu \mathrm{m}$ long, and three short terminal setae. Three caudal glands and well-developed spinneret (Fig. 4C).
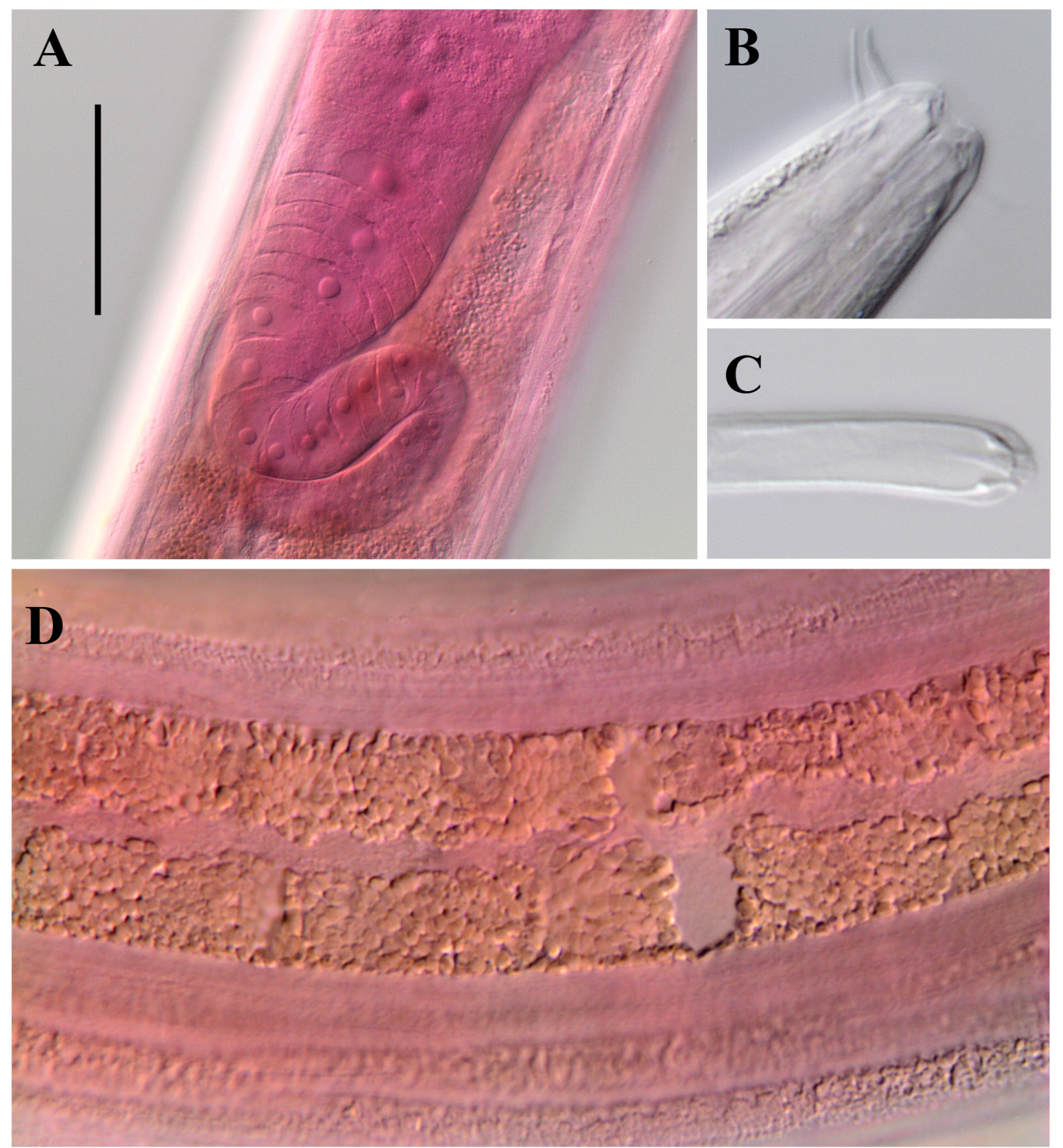

Fig. 4. Cervonema kaikouraensis sp. nov. light micrographs. A. Anterior ovary, showing bent germinal portion. B. $\widehat{\delta}$ head, showing jointed outer labial seta. C. $\delta$ spinneret. D. Granules in the lateral chords of $\hat{\delta}$. Scale bar: $\mathrm{A}=40 \mu \mathrm{m} ; \mathrm{B}=18 \mu \mathrm{m} ; \mathrm{C}=12 \mu \mathrm{m} ; \mathrm{D}=24 \mu \mathrm{m}$. 


\section{Females}

Similar to males, but with slightly larger maximum body diameter, amphideal fovea smaller, 4.5 turns. Reproductive system didelphic, opposed, with anterior branch to left of intestine and posterior branch to right of intestine. Terminal (i.e., distal) portion of anterior ovary bent in one paratype specimen (Fig. 4A), but not other paratype specimen; germinal portion of posterior ovary bent in both paratypes. Both types of sperm observed in uterus and spermathecae. Vulva at body median. Granular vaginal glands present, pars proximalis vaginae surrounded by constrictor muscle.

\section{Diagnosis and relationships}

Cervonema kaikouraensis sp. nov. is characterised by body length $1636 \mu \mathrm{m}$, amphid with 5.5 turns situated 1.7 head diameter from anterior end, jointed outer labial setae, equal in length to cephalic setae, anterior testis with large elongated sperm cells without nuclei, posterior testis with smaller nucleated sperm cells, 5 small pre-cloacal supplements, straight spicules $32 \mu \mathrm{m}$ in length, and tail $5.5 \mathrm{abd}$.

C. kaikouraensis sp. nov. can be differentiated from all other species of the genus, except $C$. allometricum Wieser, 1954 and C. pseudodeltensis Barnes et al., 2012 by the presence of jointed outer labial setae. The presence of jointed outer labial setae in C. allometricum was not described in the original description of Wieser (1954) but was later noted by Lorenzen (1981). C. kaikouraensis sp. nov. can be distinguished from $C$. allometricum and C. pseudodeltensis by the absence of gubernaculum and gubernacular apophyses (gubernaculum with conspicuous posterior apophyses present in C. allometricum and $C$. pseudodeltensis).

The presence of jointed outer labial setae may have been overlooked in previous descriptions and may not be a reliable trait to use for differentiating between all species of Cervonema. C. kaikouraensis sp. nov. can further be differentiated from most other species of the genus (except $C$. deltensis Hope \& Zhang, 1995 and C. papillatum Jensen, 1988 by the presence of sperm dimorphism. C. kaikouraensis sp. nov. differs from $C$. deltensis in body length (1636 vs. 1201-1237), length of cephalic setae (6 vs. 3-5 $\mu \mathrm{m}$ ), amphideal fovea turns (5.5 vs. 3-5), position of amphid (1.7 vs. 1.4 hd from anterior), and absence of gubernaculum (plate-like gubernaculum in C. deltensis). C. kaikouraensis sp. nov. differs from C. papillatum in body length (1636 vs. 1140-1230), and by the absence of a weakly cuticularised cap surrounding the spicules proximally. The presence or absence of sperm dimorphism was not noted in the descriptions of C. brevicauda Gourbault, 1980 and C. jenseni Gourbault, 1980, but these species differ most notably from $C$. kaikouraensis sp. nov. in the presence of a gubernaculum (absent in $C$. kaikouraensis sp. nov.). C. brevicauda also has a shorter body length (800-1090 vs. 1636), shorter cephalic setae (3 vs. $6 \mu \mathrm{m}$ ), and shorter spicules ( 0.6 vs. 0.9 abd) than $C$. kaikouraensis sp. nov. $C$. jenseni can also be differentiated from $C$. kakouraensis sp. nov. by the greater number of amphid turns (7 vs. 5.5) and absence of supplements (5 in C. kaikouraensis sp. nov.).

\section{Remarks}

The presence of bent ovaries, as observed in C. kaikouraensis sp. nov., is unusual for the family Comesomatidae. The ovaries, however, are bent only in the terminal portion. Because the ovaries in both female specimens are quite large and occupy most of the available space in the pseudocoel (Fig. 3D), this trait is considered to be a secondary feature resulting from lack of space, rather than true reflexed ovaries. 


\section{Cervonema multispira sp. nov. urn:lsid:zoobank.org:act:D4A82AED-213B-4CE4-9D62-FAAC2D44CBC0}

Figs 5-6, Table 2

\section{Etymology}

The species name is derived from the Latin multus (= much) and spiralis (= coil), and refers to the shape of the amphid.

\section{Material examined}

\section{Holotype}

\$, collected 6 Apr. 2007, southern flank of Chatham Rise (1240 m water depth), $44.4864^{\circ} \mathrm{S}, 177.1407^{\circ} \mathrm{E}$. Characteristics of surface sediment layer $(0-5 \mathrm{~mm})$ : mostly silt/clay $(82.9 \%)$, with very fine sand (10.2\%), and fine sand (8.4\%); calcium carbonate content: $38.4 \%$; total organic matter content: $1.9 \%$; chloroplastic pigment concentration: $3501 \mathrm{ng} / \mathrm{gDW}_{\text {sediment }}$ (NIC 84444).

\section{Paratypes}

$1 \hat{\jmath}, 2$ q 9 , same data as holotype (NIC 84445).

\section{Description}

\section{Males}

Body cylindrical, tapering towards both extremities, orange-brown colouration throughout. Cuticle faintly striated, no lateral differentiation. Somatic setae short and sparse. Head set-off by a depression at level of cephalic setae. Inner labial papillae indistinct, six outer labial setae, and four slightly shorter cephalic setae, the latter two in separate circles. Amphideal fovea spiral, 8.0-8.5 turns, situated 2.64.0 head diameters from anterior. Small buccal cavity, cup-shaped. Pharynx with small posterior bulb. Cardia short. Nerve ring near $60 \%$ of pharynx length from anterior. Secretory-excretory pore slightly posterior to nerve ring. Cellular body of ventral gland small, posterior to cardia. Six large uninucleated pseudocoelomocytes, $23 \mu \mathrm{m}$ wide, 35-40 $\mu \mathrm{m}$ long, in anterior half of body, with 2 and 4 cells on either side of intestine in holotype, 3 cells on either side of intestine in paratype, each with single extension (or possibly duct) directed anteriorly (Fig. 6A). Intestine wall consisting mostly of cells containing small granules, interspersed with clusters of clear globular inclusions

Reproductive system diorchic, opposed, outstretched. Anterior testis to left of intestine, posterior testis to right of intestine. Sperm cells small (up to $5 \times 4 \mu \mathrm{m}$ ), globular, with lenticular nuclei situated peripherally. Spicules paired, equal, arcuate, 0.9-1.1 abd long, strongly cuticularised along entire length, with capitulum. Four pairs of small ejaculatory glands situated anteriorly to spicules (Fig. 5C). Small rectal gland present. Gubernaculum not observed. Five small, tubular pre-cloacal supplements, one precloacal seta. Tail conico-cylindrical, with several short caudal setae and three short terminal setae. Three caudal glands and spinneret present.

\section{Females}

Similar to males, but with smaller amphideal fovea, 7.5 turns. Large nucleated cells in anterior part of body not observed. Reproductive system didelphic, opposed, outstretched, with anterior branch to left of intestine and posterior branch to right of intestine. Spermatheca present, containing sperm. Vulva slightly post median. Granular vaginal glands present, pars proximalis vaginae surrounded by constrictor muscle. 


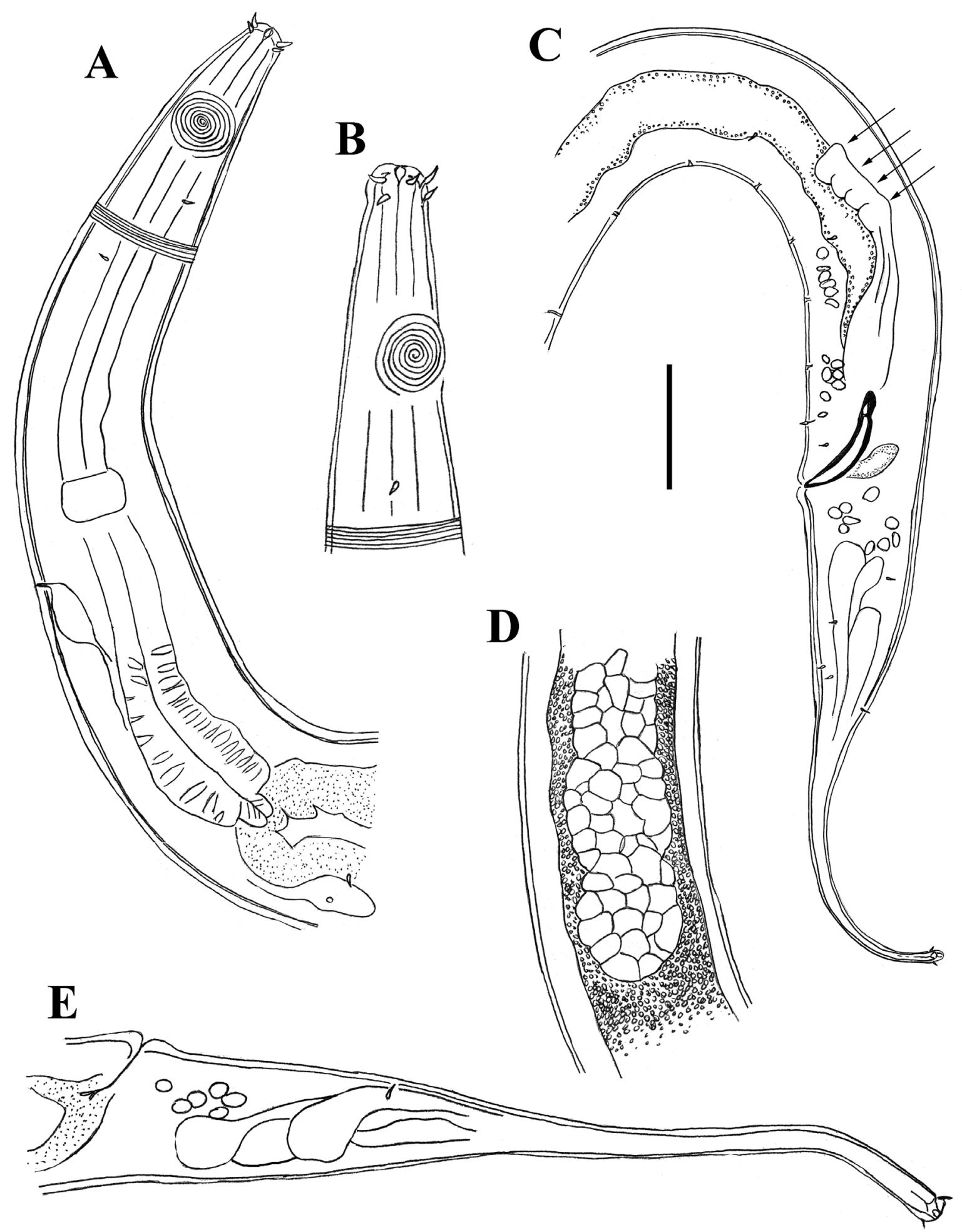

Fig. 5. Cervonema multispira sp. nov. A. $\widehat{\partial}$ anterior body region. B. $q$ head. C. $\widehat{\partial}$ posterior body region, showing copulatory apparatus. D. $q$ intestine. E. $q$ tail. Arrows point to the four ejaculatory glands. Scale bar: $\mathrm{A}=30 \mu \mathrm{m} ; \mathrm{B}=20 \mu \mathrm{m} ; \mathrm{C}=40 \mu \mathrm{m} ; \mathrm{D}=28 \mu \mathrm{m} ; \mathrm{E}=25 \mu \mathrm{m}$. 


\section{Diagnosis and relationships}

Cervonema multispira sp. nov. is characterised by body length $1225-1431 \mu \mathrm{m}$, amphideal fovea with 8.0-8.5 turns, 2.6-4.0 head diameter from anterior end, cephalic setae 2-3 $\mu \mathrm{m}$ long, slightly shorter than outer labial setae, presence of six large lateral cells in males only, 5 small pre-cloacal supplements, strongly cuticularised, arcuate spicules with capitulum, and tail 4.4-6.1 abd.

Cervonema multispira sp. nov. resembles C. macramphis Jensen, 1979 in body size, length of cephalic setae, amphid with numerous ( $>7)$ turns, and the presence of arcuate spicules. Cervonema multispira sp. nov. can be differentiated from C. macramphis and all other species of the genus by amphideal fovea with 8.0-8.5 turns $(<7.5$ turns in all other species), presence of lateral excretory cells in males, and strongly cuticularised spicules with capitulum. C. multipsira sp. nov. also resembles $C$. pseudodeltensis Barnes et al., 2012 in having outer labial setae marginally longer than cephalic setae, but is characterised by shorter body length (1225-1431 vs. 1559-1755 $\mu \mathrm{m})$, amphideal fovea with more turns (8.0-8.5 vs. 5.5) and absence of gubernaculum (present in C. pseudodeltensis).

\section{Discussion}

To my knowledge, this is the first time that the presence of large uninucleated cells is noted in the pseudocoelom of Cervonema specimens. Similar cells, usually situated laterally, and sometimes subventrally, have been described in Sabatieria celtica Southern, 1914 (Riemann 1977), S. pulchra (Schneider, 1906) (Jensen 1979), S. breviseta Stekhoven, 1935, and S. punctata (Kreis, 1924) (Platt
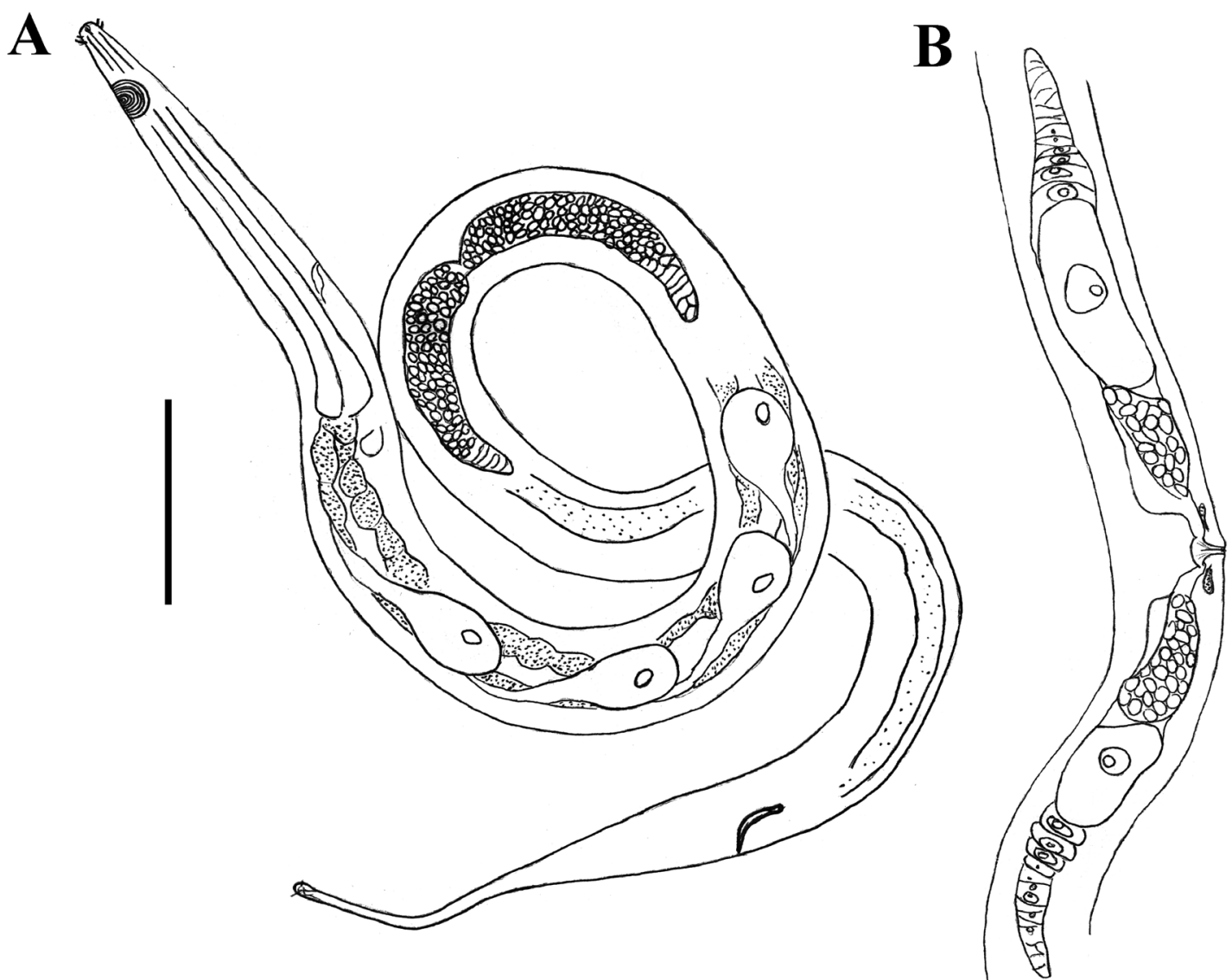

Fig. 6. Cervonema multispira sp. nov. A. Entire $\delta^{\lambda}$. B. + reproductive system. Scale bar: A $=100 \mu \mathrm{m}$; $\mathrm{B}=70 \mu \mathrm{m}$. 
Table 2. Morphometrics $(\mu \mathrm{m})$ of Cervonema multipsira sp. nov. and C. proberti sp. nov. n/o = not observed.

\begin{tabular}{|c|c|c|c|c|c|c|}
\hline \multirow[t]{3}{*}{ Species } & \multicolumn{3}{|c|}{ Cervonema multispira sp. nov. } & \multicolumn{3}{|c|}{ Cervonema proberti sp. nov. } \\
\hline & \multicolumn{2}{|c|}{ Males } & \multirow{2}{*}{$\begin{array}{c}\text { Females } \\
\text { Paratypes }\end{array}$} & \multicolumn{2}{|c|}{ Males } & \multirow{2}{*}{$\begin{array}{c}\text { Females } \\
\text { Paratypes }\end{array}$} \\
\hline & Holotype & Paratype & & Holotype & Paratype & \\
\hline $\mathbf{n}$ & & 1 & 2 & & 1 & 3 \\
\hline $\mathbf{L}$ & 1431 & 1225 & $965-1083$ & 1144 & 1043 & $1183-1397$ \\
\hline $\mathbf{a}$ & 28 & 34 & $24-29$ & 30 & 33 & $33-35$ \\
\hline b & 7 & 7 & $6-7$ & 9 & 9 & $9-10$ \\
\hline c & 9 & 7 & $7-13$ & 9 & 9 & 8 \\
\hline Head diam. & 10 & 9 & 8 & 12 & 10 & $11-12$ \\
\hline Mouth diam. & 2 & 2 & 2 & 1 & $<1$ & $1-2$ \\
\hline Length of cephalic setae & 3 & 2 & 2 & 7 & $7-8$ & $8-9$ \\
\hline Amphid height & 15 & 14 & $10-11$ & 13 & 14 & 10 \\
\hline Amphid width & 16 & 14 & $9-10$ & 11 & 12 & $9-10$ \\
\hline Amphid width/cbd (\%) & 76 & 82 & $69-71$ & 85 & 86 & $69-71$ \\
\hline Amphid from anterior end & 40 & 23 & $19-22$ & 6 & 10 & $9-10$ \\
\hline Nerve ring from anterior end & 125 & 108 & $90-108$ & 68 & 64 & $65-67$ \\
\hline Nerve ring cbd & 38 & 28 & $25-27$ & 28 & 25 & $28-29$ \\
\hline Excretory pore from anterior end & 147 & 123 & $113-126$ & $\mathrm{n} / \mathrm{o}$ & $\mathrm{n} / \mathrm{o}$ & $87-90$ \\
\hline Pharynx length & 214 & 183 & 151-185 & 129 & 120 & $131-144$ \\
\hline Pharynx cbd & 47 & 35 & $30-32$ & 34 & 28 & $32-35$ \\
\hline Pharyngeal bulb diam. & 26 & 20 & $19-20$ & 26 & 18 & $25-27$ \\
\hline Max. body diam. & 51 & 36 & $40-42$ & 38 & 32 & $36-42$ \\
\hline Spicule length & 36 & 31 & - & 20 & 22 & - \\
\hline Gubernacular apophyses length & - & - & - & - & - & - \\
\hline Anal body diam. & 38 & 29 & $26-27$ & 27 & 24 & $26-30$ \\
\hline Tail length & 167 & 176 & $146-147$ & 122 & 116 & $142-178$ \\
\hline Tail length/abd & 4.4 & 6.1 & $5.4-5.6$ & 4.5 & 4.8 & $5.5-5.9$ \\
\hline $\mathbf{V}$ & - & - & $488-628$ & - & - & $556-644$ \\
\hline$\% \mathrm{~V}$ & - & - & $51-52$ & - & - & $46-47$ \\
\hline Vulval body diam. & - & - & $38-42$ & - & - & $36-42$ \\
\hline
\end{tabular}

1984). Only two cells (described as supplementary excretory cells) are found in Sabatieria spp., whereas six were observed in Cervonema multispira sp. nov. This type of cell has not been observed in females of Sabatieria spp. or C. multispira sp. nov.

Cervonema proberti sp. nov.

urn:1sid:zoobank.org:act:0635B2CB-1114-4399-A3E6-DC0F53832755

Figs 7-8, Table 2

\section{Etymology}

This species is named after Dr. P. Keith Probert. 


\section{Material examined}

\section{Holotype}

${ }^{\top}$, collected 5 Oct. 2001 , Chatham Rise crest $\left(350 \mathrm{~m}\right.$ water depth), $43.4333^{\circ} \mathrm{S}, 178.5000^{\circ} \mathrm{E}$. Characteristics of surface sediment layer $(0-5 \mathrm{~mm})$ : mostly fine sand $(35.7 \%)$, silt/clay $(33.0 \%)$, and very fine sand (23.5\%); calcium carbonate content: $40.5 \%$; total organic matter content: $4.5 \%$; chloroplastic pigment concentration: $3359 \mathrm{ng} / \mathrm{gDW}_{\text {sediment }}$ (NIC 84442).

\section{Paratypes}

1 §, 3 우, same data as holotype (NIC 84443).

\section{Description}

\section{Males}

Body cylindrical, tapering towards both extremities. Cuticle faintly striated, no lateral differentiation. Somatic setae short and sparse, in four dorso- and ventro-lateral longitudinal rows. Epidermal glands associated with somatic setae visible as clear, oval areas underneath cuticle (Figs 7C, 8A). Head set-off by constriction at level of amphids. Inner labial papillae indistinct, six outer labial setae, and four longer cephalic setae, the latter two almost at same level. Cephalic setae 1.6-2.0 times longer than outer labial setae. Amphideal fovea spiral, 5 turns, 0.5-1.0 head diameter from anterior. Small buccal cavity, cupshaped. Pharynx with rounded or oval terminal bulb. Nucleus of dorsal pharyngeal gland conspicuous. Cardia short. Nerve ring near middle of pharynx length. Secretory-excretory pore slightly posterior to nerve ring. Cellular body of ventral gland slightly posterior to cardia. Intestine wall consisting mostly of cells containing colourless granules, interspersed with clusters of clear globular inclusions (Fig. 8B).

Reproductive system diorchic, opposed, outstretched. Anterior testis to left of intestine, posterior testis to right of intestine. Oval-shaped sperm cells with lenticular nuclei situated peripherally (Fig. 7D). Two pairs of ejaculatory glands present. Spicules paired, equal, slightly arcuate, 0.7-0.9 abd long, proximal end slightly cephalated, gradually tapering towards distal end. Gubernaculum not observed. Rectal gland surrounds spicules. Eight small, tubular pre-cloacal supplements, often difficult to distinguish, one precloacal seta. Tail conico-cylindrical, with several short caudal setae and three short terminal setae. Three caudal glands and spinneret present.

\section{Females}

Similar to males, but with slightly larger maximum body diameter, amphideal fovea smaller, 4-5 turns, slightly longer tail. Reproductive system didelphic, opposed, outstretched, with anterior branch to left of intestine and posterior branch to right of intestine. Vulva slightly pre median. Granular vaginal glands present, pars proximalis vaginae surrounded by constrictor muscle.

\section{Diagnosis and relationships}

Cervonema proberti sp. nov. is characterised by body length 1043-1144 $\mu \mathrm{m}$, amphideal fovea with 5 turns and $<1$ head diameter from anterior end, cephalic setae 1.6-2.0 times longer than outer labial setae, sperm cells with lenticular nuclei, 8 small pre-cloacal supplements, and tail 4.5-5.9 abd.

Cervonema proberti sp. nov. can be distinguished from most other species of the genus by the amphid $<1$ head diameter from anterior, compared to $>1$ head diameter from anterior in most species (except C. hermani Chen \& Vincx, 2000, C. chilensis Chen \& Vincx, 2000, and C. proximamphidum Tchesunov, 2000). C. proberti sp. nov. can be distinguished from C. hermani, C. chilensis, and C. proximamphidum by cephalic setae conspicuously (1.6-2.0×) longer than outer labial setae (almost equal in length in $C$. hermani, C. chilensis, and C. proximamphidum), and nucleated sperm cells (no nuclei in C. hermani, C. 


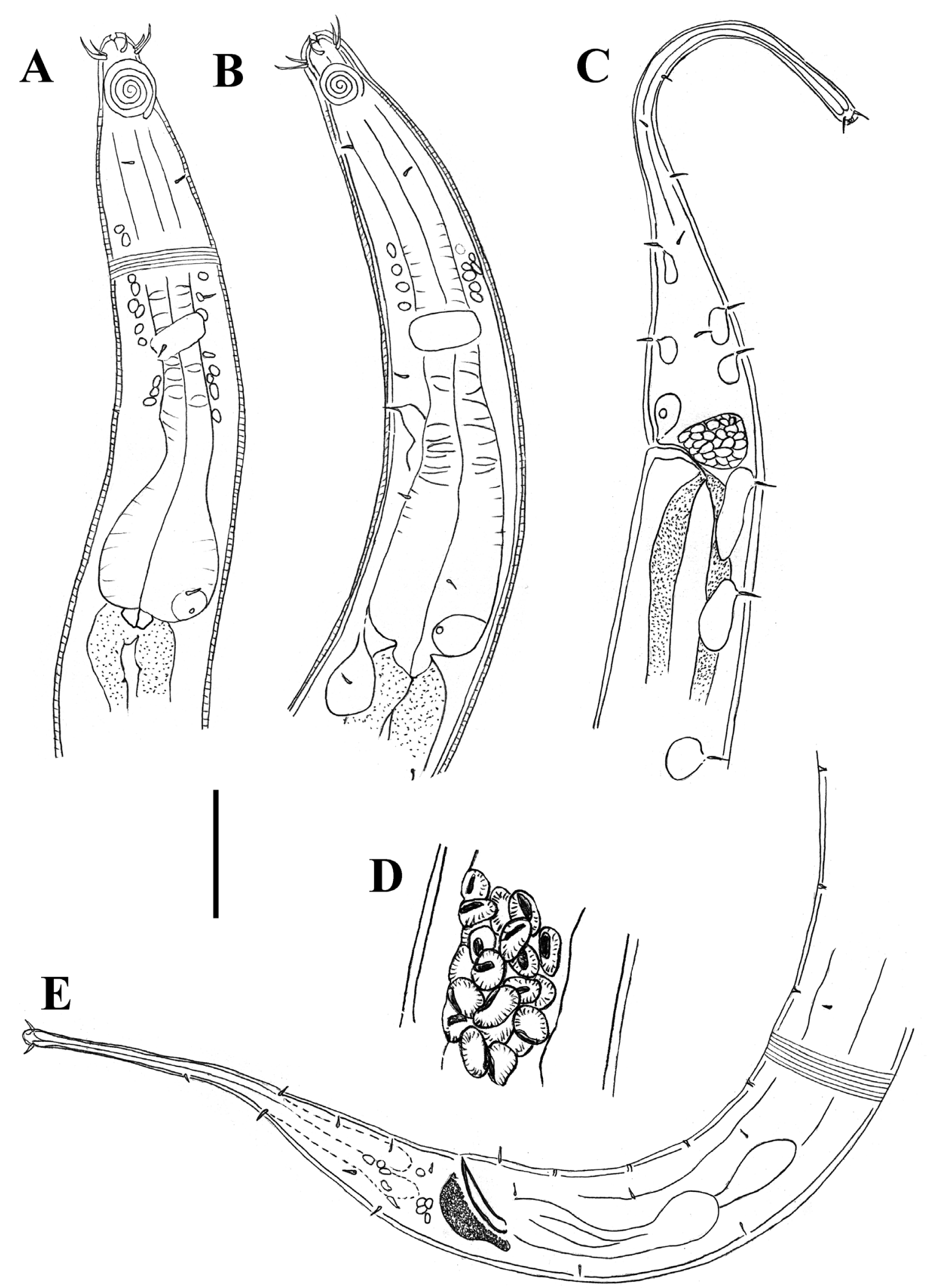

Fig. 7. Cervonema proberti sp. nov. A. $\lesssim$ anterior body region. B. $q$ anterior region. C. $q$ tail. D. Sperm cells. E. $\widehat{O}$ posterior body region, showing copulatory apparatus. Scale bar: A, B, and C $=25 \mu \mathrm{m}$; $\mathrm{D}=20 \mu \mathrm{m} ; \mathrm{E}=35 \mu \mathrm{m}$. 
chilensis, and C. proximamphidum). C. proberti sp. nov. is also characterised by a shorter tail than $C$. hermani (4.5-4.8 vs. 5.7-9.3 abd). C. proberti sp. nov. resembles Cervonema sp. B described by Barnes et al. (2012) based on one female specimen from the Korean west coast. My female specimens share several traits, including amphid close to anterior end, well-developed pharyngeal bulb with conspicuous pharyngeal gland nucleus, and cephalic setae longer than the outer labial setae. My specimens, however, have shorter body length (1183-1397 vs. $1727 \mu \mathrm{m})$, and greater ratio of cephalic setae length to outer labial setae length (1.6-2.0 vs. 1.3). Male specimens are required before the identity of the Korean species can be determined with certainty.

A

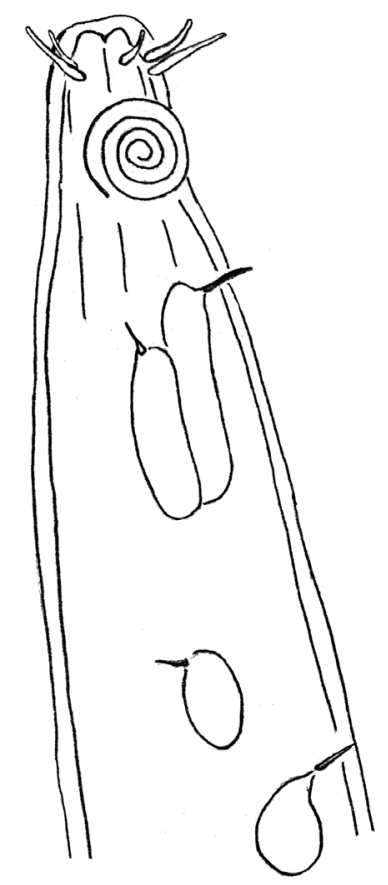

C

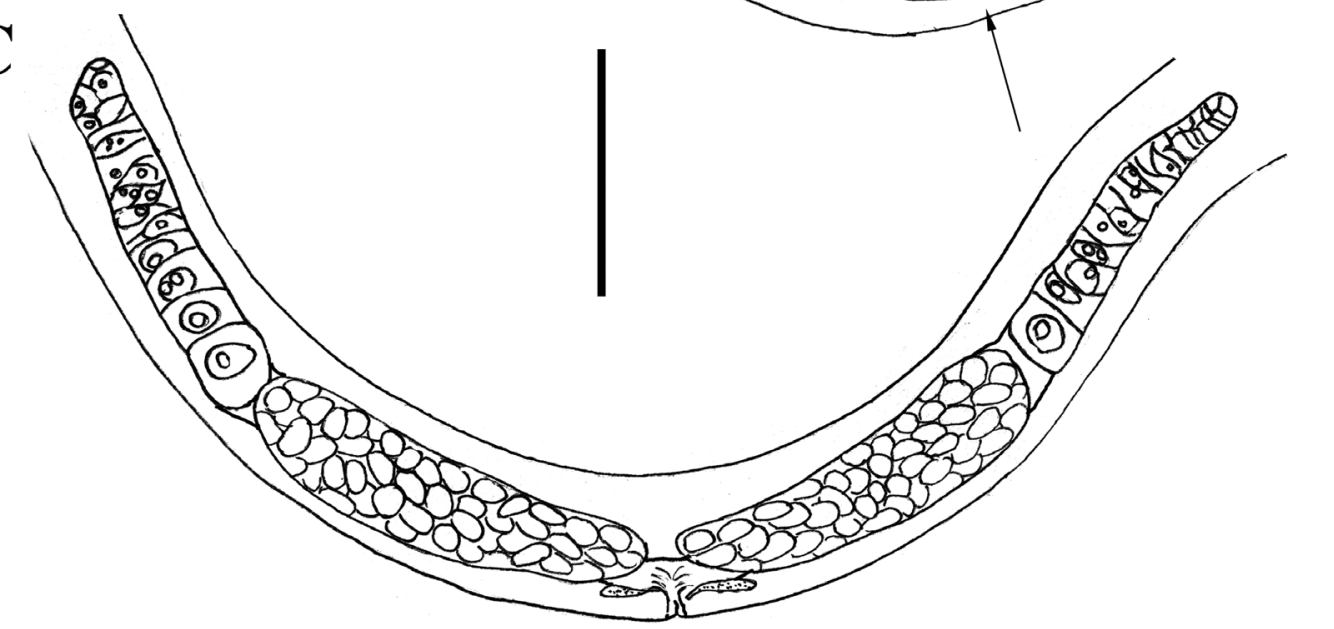

Fig. 8. Cervonema proberti sp. nov. A. $q$ head, showing glands. B. Entire $\lesssim$. C. $q$ reproductive system. Arrows point to clear globular inclusions in intestine wall. Scale bar: $\mathrm{A}=20 \mu \mathrm{m} ; \mathrm{B}$ and $\mathrm{C}=65 \mu \mathrm{m}$. 
Table 3. Male morphological characters of all known Cervonema species.

\begin{tabular}{|c|c|c|c|c|c|c|c|c|c|c|c|c|c|c|c|c|c|c|}
\hline 胥 总 & $\begin{array}{l}0 \\
\dot{1} \\
1 \\
n \\
n \\
n\end{array}$ & $\begin{array}{l}\stackrel{0}{+} \\
\stackrel{+}{+} \\
\stackrel{r}{r}\end{array}$ & in & $\begin{array}{l}0 \\
\dot{0} \\
0 \\
\dot{1} \\
\text {. }\end{array}$ & $\stackrel{i}{i}$ & $\begin{array}{l}n \\
0 \\
0 \\
0 \\
0 \\
0\end{array}$ & $\begin{array}{l}m \\
a \\
i \dot{i} \\
i\end{array}$ & $\begin{array}{l}0 \\
\dot{0} \\
0 \\
\dot{1} \\
r\end{array}$ & in & $\begin{array}{c}\overrightarrow{+} \\
\dot{+} \\
\dot{r} \\
\dot{r}\end{array}$ & $\begin{array}{l}\dot{t} \\
\dot{b} \\
b \\
\dot{b} \\
\text {. }\end{array}$ & ì & $\begin{array}{l}\vec{f} \\
b \\
\dot{b} \\
\infty \\
\dot{\sigma} \\
\dot{0}\end{array}$ & $\begin{array}{l}0 \\
\hat{i} \\
\dot{b} \\
\dot{b}\end{array}$ & ñ & $\begin{array}{l}-\overrightarrow{0} \\
\dot{j} \\
\dot{+} \\
\dot{+}\end{array}$ & $\begin{array}{l}\infty \\
\stackrel{+}{+} \\
\stackrel{+}{+} \\
+\end{array}$ & $\frac{a}{2}$ \\
\hline 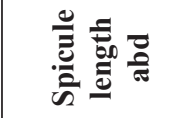 & $\stackrel{\circ}{\circ}$ & $\stackrel{0}{0}$ & $\stackrel{\circ}{\dot{\vec{v}}}$ & $\begin{array}{l}0 \\
\dot{0} \\
0 \\
0 \\
0\end{array}$ & gे & $\grave{0}$ & $\stackrel{\circ}{-}$ & 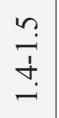 & $\hat{0}$ & $\begin{array}{l}0 \\
\stackrel{\dot{b}}{-} \\
\stackrel{\infty}{0} \\
\dot{0}\end{array}$ & $\begin{array}{l}0 \\
\stackrel{1}{1} \\
\dot{\infty} \\
0 \\
0\end{array}$ & $\mid$\begin{tabular}{l|}
0 \\
0 \\
0 \\
$o$ \\
0 \\
0
\end{tabular} & $\begin{array}{l}0 \\
\dot{1} \\
\dot{b} \\
0 \\
0\end{array}$ & $\stackrel{\circ}{-}$ & 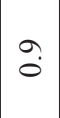 & $\frac{7}{8}$ & 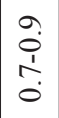 & $\begin{array}{l}\tilde{N} \\
\infty\end{array}$ \\
\hline 竞恶 & $\ddot{\nu}$ & $\stackrel{\infty}{\infty}$ & $\stackrel{\circ}{z}$ & 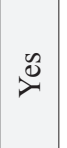 & $\ddot{z}$ & $\stackrel{0}{0}$ & 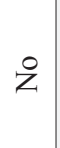 & 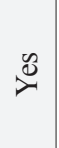 & $\stackrel{2}{z}$ & $\ddot{z}$ & $\stackrel{\varpi}{\nu}$ & $\stackrel{\circ}{z}$ & z & $\stackrel{\infty}{\infty}$ & z & $\stackrel{\circ}{z}$ & z & 莃 \\
\hline 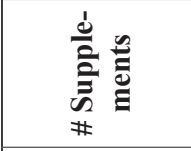 & $\tilde{Z}$ & $\tilde{Z}^{\infty}$ & $r$ & $r$ & 0 & 0 & $a$ & 0 & 0 & $\hat{b}$ & in & $\frac{0}{\alpha}$ & $\stackrel{n}{f}$ & 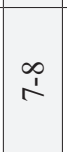 & in & in & $\infty$ & $\Xi$ \\
\hline 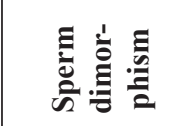 & $\bar{z}$ & $\tilde{z}$ & z & $\stackrel{0}{0}$ & ż & $\tilde{z}$ & $\stackrel{\circ}{z}$ & $\stackrel{\circ}{z}$ & $\stackrel{0}{z}$ & $\begin{array}{l}\hat{y} \\
\bar{y}\end{array}$ & 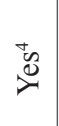 & ż & $\tilde{z}$ & $\stackrel{\circ}{z}$ & $\sum_{i}^{\infty}$ & z & z & 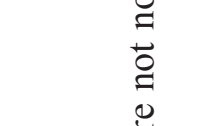 \\
\hline 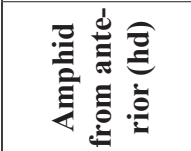 & 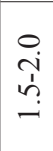 & $\stackrel{\infty}{-}$ & $\stackrel{n}{\mathfrak{o}}$ & $\stackrel{\Xi}{-}$ & $\lambda$ & $\stackrel{\bullet}{-}$ & $\stackrel{n}{\hat{0}}$ & $\stackrel{\leftrightarrow}{i}$ & $\begin{array}{c}\dot{\lambda} \\
\dot{\lambda}\end{array}$ & $\stackrel{\circ}{i}$ & $\stackrel{i}{\dot{s}}$ & $\begin{array}{l}\infty \\
0 \\
0\end{array}$ & 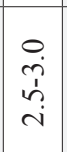 & $\stackrel{\circ}{i}$ & 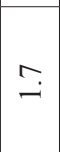 & $\begin{array}{l}0 \\
\dot{+} \\
b \\
i \\
i\end{array}$ & $\frac{0}{\dot{1}}$ & 弚 \\
\hline 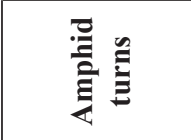 & $\begin{array}{l}0 \\
\dot{r} \\
\dot{n} \\
\dot{\gamma}\end{array}$ & $\begin{array}{l}0 \\
\dot{b} \\
\dot{n} \\
i n \\
\text { in }\end{array}$ & $n$ & $\stackrel{n}{n}$ & $n$ & $r$ & 盀 & $\stackrel{0}{\circ}$ & $\mathfrak{q}$ & 0 & in & $\begin{array}{l}0 \\
\dot{1} \\
n\end{array}$ & \begin{tabular}{|l|}
$n$ \\
0 \\
0 \\
0 \\
$i n$ \\
$n$
\end{tabular} & $\begin{array}{l}0 \\
b \\
\dot{1} \\
i n \\
i n\end{array}$ & 盀 & $\mid \begin{array}{c}n \\
\infty \\
0 \\
0 \\
\infty \\
\infty\end{array}$ & in & 造 \\
\hline 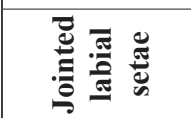 & 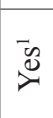 & $\ddot{z}$ & z & ̊̃ & $\stackrel{\circ}{z}$ & $\ddot{z}$ & $\dot{z}$ & $\dot{z}$ & $\dot{z}$ & $\dot{z}$ & 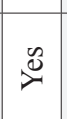 & ż & ż & $\dot{z}$ & $\stackrel{\infty}{\infty}$ & z & z & $\frac{\pi}{\pi}$ \\
\hline 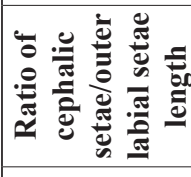 & $\stackrel{?}{-}$ & $\stackrel{0}{i}$ & $\stackrel{0}{-}$ & $\stackrel{\circ}{-}$ & $\stackrel{\circ}{-}$ & $\stackrel{\circ}{-}$ & $\stackrel{\leftrightarrow}{-}$ & $\stackrel{-}{-}$ & $\stackrel{-}{-}$ & $\stackrel{\circ}{-}$ & $\left|\begin{array}{l}\infty \\
0 \\
0 \\
0 \\
0 \\
0\end{array}\right|$ & $\stackrel{\circ}{-}$ & $\stackrel{?}{-}$ & $\stackrel{?}{-}$ & $\stackrel{\circ}{-}$ & $\frac{0}{\frac{1}{n}}$ & 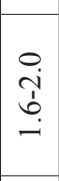 & 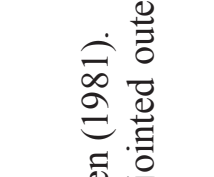 \\
\hline 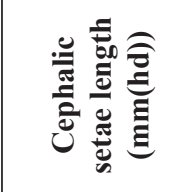 & $\begin{array}{l}\hat{\sigma} \\
\dot{1} \\
0 \\
e \\
e \\
a\end{array}$ & 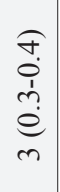 & 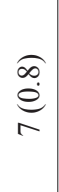 & $\begin{array}{c}n \\
n \\
o \\
+ \\
+ \\
e \\
n \\
n \\
m\end{array}$ & $\begin{array}{l}\frac{f}{d} \\
\stackrel{e}{a} \\
q \\
f\end{array}$ & $\begin{array}{c}\stackrel{f}{d} \\
\stackrel{d}{\sigma}\end{array}$ & $\mid \begin{array}{c}0 \\
0 \\
0 \\
0 \\
\vdots \\
\infty \\
\infty\end{array}$ & $\stackrel{\substack{e \\
e}}{e}$ & 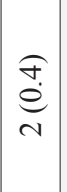 & 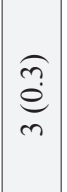 & 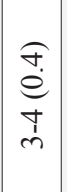 & $\begin{array}{c}0 \\
0 \\
0 \\
n \\
n \\
0 \\
0 \\
i \\
\end{array}$ & 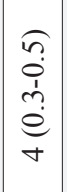 & $\begin{array}{c}n \\
\hat{n} \\
e \\
+\end{array}$ & $\begin{array}{l}6 \\
\stackrel{6}{0} \\
0\end{array}$ & 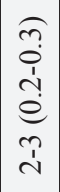 & $\begin{array}{l}0 \\
0 \\
0 \\
b \\
0 \\
e \\
\infty \\
1 \\
1\end{array}$ & 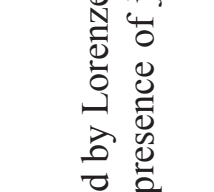 \\
\hline 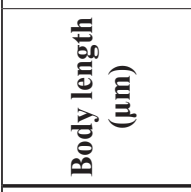 & $\stackrel{8}{0}$ & $\begin{array}{l}0 \\
8 \\
0 \\
\vdots \\
8 \\
\infty\end{array}$ & $\underset{\Xi}{\Xi}$ & $\begin{array}{l}\hat{\tilde{a}} \\
\frac{i}{\grave{a}} \\
\end{array}$ & 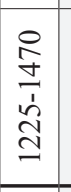 & 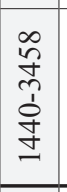 & 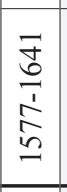 & $\begin{array}{l}\stackrel{g}{0} \\
-1\end{array}$ & $\begin{array}{c}m \\
\hat{\infty} \\
0 \\
0 \\
n \\
n\end{array}$ & 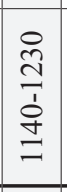 & 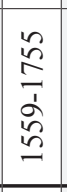 & 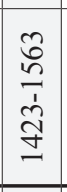 & 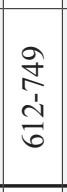 & 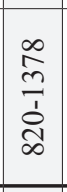 & $\begin{array}{l}0 \\
0 \\
0 \\
-\end{array}$ & 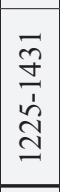 & 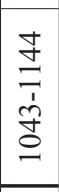 & 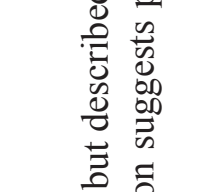 \\
\hline $\begin{array}{l}\frac{\mathscr{c}}{\tilde{u}} \\
\text { की }\end{array}$ & 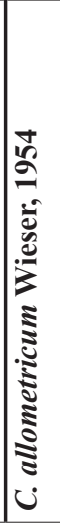 & 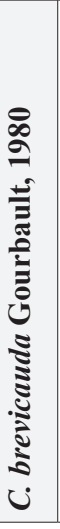 & 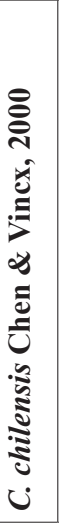 & 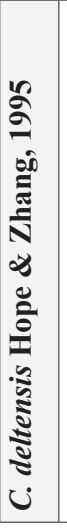 & 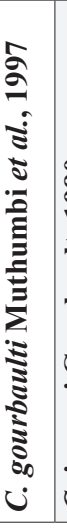 & 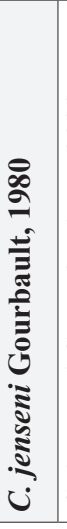 & 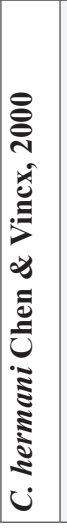 & 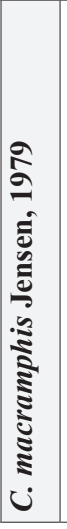 & 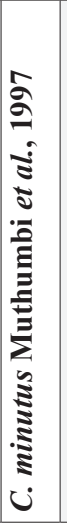 & 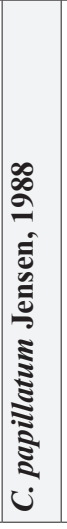 & 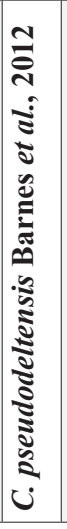 & 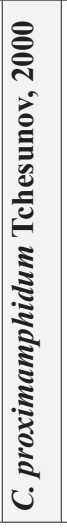 & 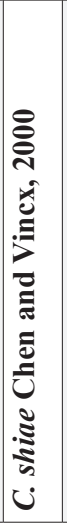 & 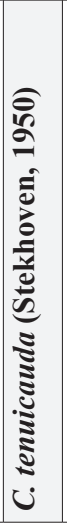 & 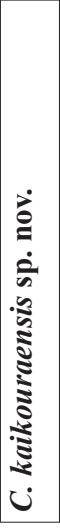 & 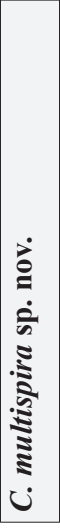 & 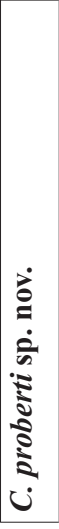 & 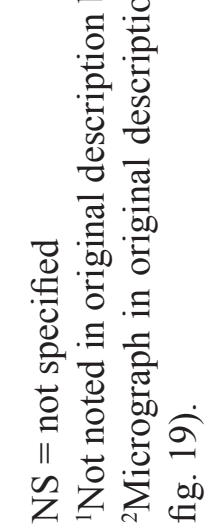 \\
\hline
\end{tabular}




\section{Discussion}

A total of 17 Cervonema species (including the three new species described herein) have been described so far. Differentiating between Cervonema species can be difficult due to relatively subtle differences in morphology. A summary of key morphological features of all known Cervonema species is provided to help identification (Table 3).

\section{Subfamily Dorylaimopsinae De Coninck, 1965}

\section{Diagnosis (modified from Jensen (1979))}

Cuticle usually differentiated laterally. Cephalic sense organs in three distinctly separated crowns. Buccal cavity usually strongly cuticularised; anterior portion shallow, sometimes with small pointed projections at border with vestibulum; posterior portion dilated, cylindrical or conical, usually with strongly cuticularised walls and three thornlike projections (teeth) at the border between the two portions. Spicules sometimes differentiated proximally, medially or distally. Gubernacular apophyses usually directed caudally, occasionally dorsocaudally.

\section{Type genus}

Dorylaimopsis Ditlevsen, 1918

\section{Remarks}

The genus Dorylaimopsis is similar in many respects to the genus Hopperia, but can be distinguished from the latter by the presence of longitudinal rows of dots as opposed to larger, irregularly-distributed dots in the lateral fields (sometimes arranged in transverse rows) in Hopperia. There are two exceptions: (1) some specimens of $H$. communis Gagarin \& Thanh, 2006 have irregularly distributed dots in the lateral fields, and (2) D. metatypica Chitwood, 1936 is characterised by irregularly-spaced dots instead of longitudial rows. The two genera also tend to differ in the shape of the spicules: arcuate in Hopperia, whilst in Dorylaimopsis spicules may be arcuate (D. coomansi Muthumbi et al., 1997), jointed (D. perfecta Cobb, 1920), or with lateral projections (D. punctata Ditlevsen, 1918). In addition, species of the genus Dorylaimopsis are characterised by conico-cylindrical tail with (usually) swollen tip, whilst tail shape in Hopperia can be conico-cylindrical with swollen (H. americana Pastor de Ward, 1984), filiform (H. massiliensis Vitiello, 1969), or pointed tip (H. australis Jensen, 1992).

\section{Genus Dorylaimopsis Ditlevsen, 1918}

\section{Diagnosis (modified from Jensen (1979))}

Dorylaimopsinae. Cuticle with lateral differentiation consisting of longitudinal rows of coarse dots; posterior portion of buccal cavity cylindrical, with three thorn-like teeth at border to anterior portion; outer labial sensillae and cephalic setae in separate circles; spicules usually long, arcuate or jointed; caudal or dorso-caudal gubernacular apophyses.

\section{Type species}

Dorylaimopsis punctata Ditlevsen, 1918 


\section{Dorylaimopsis nodderi sp. nov. urn:1sid:zoobank.org:act:A19205F2-A989-49EA-9002-46CA34F64CCA}

Figs 9-11, Table 4

\section{Etymology}

This species is named after Dr. Scott D. Nodder.

\section{Material examined}

\section{Holotype}

${ }^{\Uparrow}$, collected 5 Oct. 2001 , Chatham Rise crest $\left(350 \mathrm{~m}\right.$ water depth), $43.4333^{\circ} \mathrm{S}, 178.5000^{\circ} \mathrm{E}$. Characteristics of surface sediment layer $(0-5 \mathrm{~mm})$ : mostly fine sand $(35.7 \%)$, silt/clay $(33.0 \%)$, and very fine sand (23.5\%); calcium carbonate content: $40.5 \%$; total organic matter content: $4.5 \%$; chloroplastic pigment concentration: $3359 \mathrm{ng} / \mathrm{gDW}_{\text {sediment }}$ (NIC 84446).

\section{Paratypes}

$4 \hat{\jmath} \hat{\jmath}, 4$ 우, same data as holotype (NIC 84447).

\section{Description}

\section{Males}

Body cylindrical, tapering slightly towards both extremities. Cuticle punctate from level of amphid to near tail tip, with lateral differentiation consisting of three longitudinal rows of larger dots in pharyngeal and caudal regions, two rows of larger dots elsewhere. Cuticle in lateral field consisting of $1 \mu \mathrm{m}$ thick basal layer, $4 \mu \mathrm{m}$ thick median layer with forked columns (side view), and $2 \mu \mathrm{m}$ thick cortical layer (Fig. 11A). Cuticle thinner in caudal region. Eight longitudinal rows of short, sparse somatic setae. Head set-off by constriction immediately posterior to cephalic setae. Six inner labial papillae, six outer labial papillae, and four cephalic setae. Anterior portion of buccal cavity cup-shaped. Posterior portion of buccal cavity cylindrical, cuticularised, narrow, 13-16 $\mu \mathrm{m}$ deep, with three cuticularised projections (teeth) at border to anterior portion. Amphideal fovea spiral, 2.5-3.0 turns, located immediately posterior to cephalic setae. Pharynx gradually swelling, not forming true bulb. Marginal tubes beginning from base of stoma. Cardia short. Nerve ring near middle of pharynx, situated anteriorly to secretory-excretory pore. Cellular body of ventral gland at level of cardia. Intestine wall with numerous small granules, interspersed with numerous regularly-spaced clear areas without granules, circular or irregular in shape (Fig. 9E).

Reproductive system diorchic, opposed, outstretched. Anterior testis to left of intestine, posterior testis to right of intestine. Spicules paired, equal, 1.8-2.9 abd long, with rounded ventral projection at one third of spicule length from distal end, giving appearance of a joint. Spicules straight proximally, strongly bent at level of joint. Gubernaculum surrounding spicules, with wide triangular base and pair of long, straight caudal apophyses. Rectal gland surrounds gubernaculum. Twelve to sixteen tubular pre-cloacal supplements. Tail conico-cylindrical, with several short caudal setae and three short terminal setae. Three caudal glands.

\section{Females}

Similar to males, but with slightly smaller amphideal fovea, 2.5-2.75 turns. Reproductive system didelphic, opposed, outstretched, with anterior branch to left of intestine and posterior branch either to right or left of intestine. Vulva slightly pre median. Granular vaginal glands present, pars proximalis vaginae surrounded by constrictor muscle. 

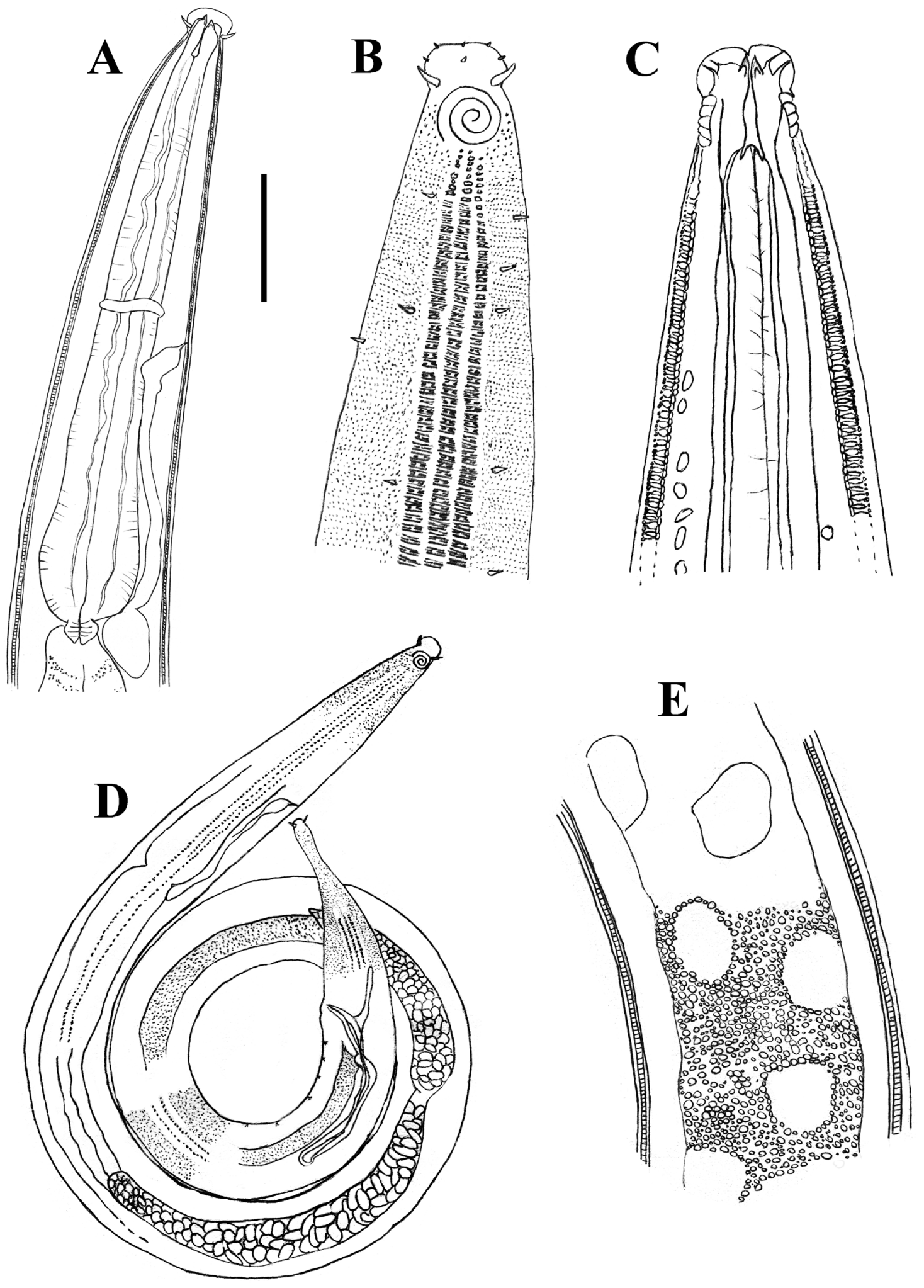

Fig. 9. Dorylaimopsis nodderi sp. nov. A. Anterior region of $\widehat{\partial}$. B. Lateral surface view of $\delta^{\lambda}$ head.

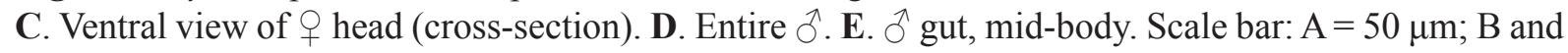
$\mathrm{C}=25 \mu \mathrm{m} ; \mathrm{D}=70 \mu \mathrm{m} ; \mathrm{E}=30 \mu \mathrm{m}$. 

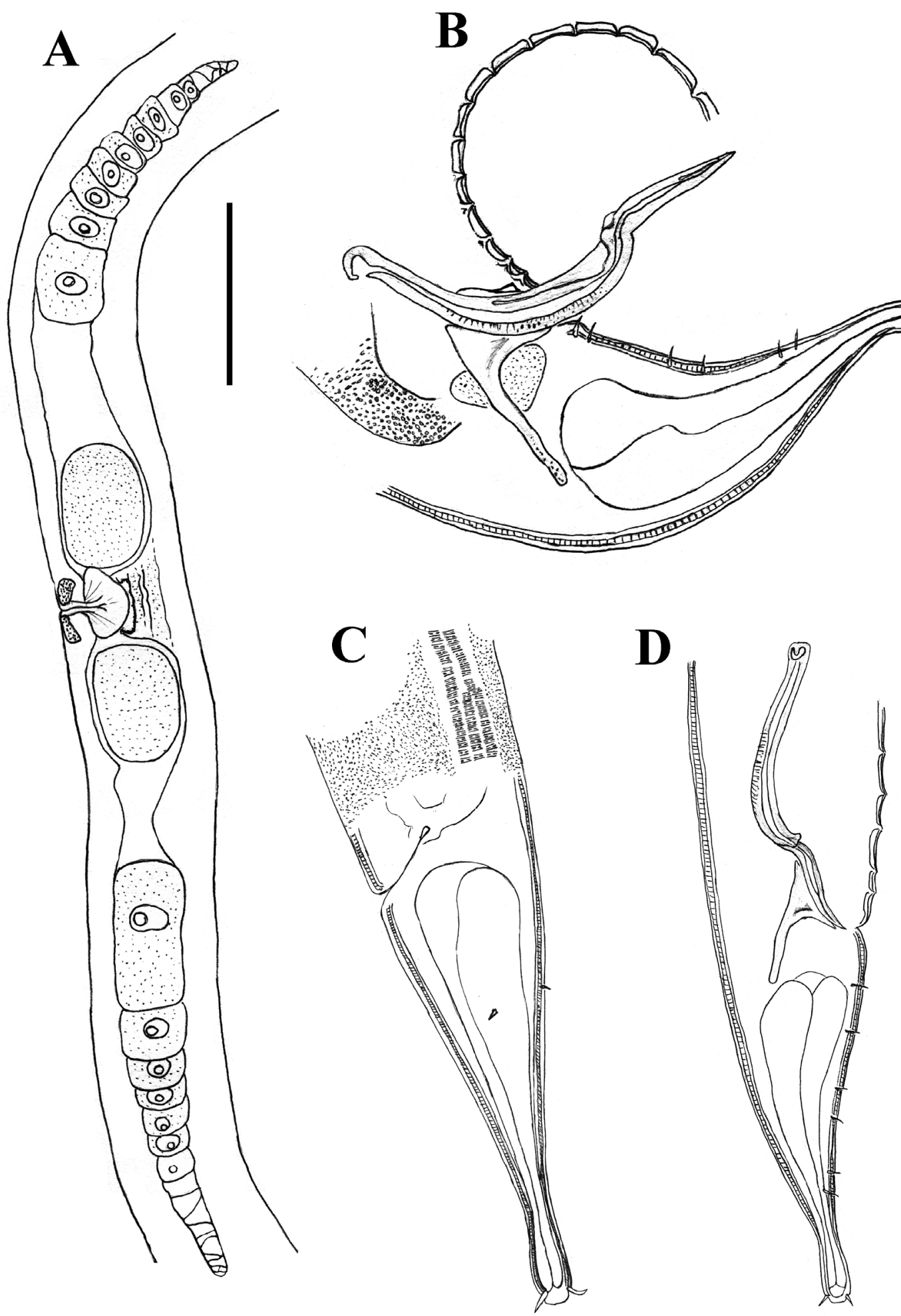

Fig. 10. Dorylaimopsis nodderi sp. nov. A. $q$ reproductive system. B. $\widehat{\jmath}$ posterior region, showing copulatory apparatus. C. + tail. D. $\delta$ posterior body region, showing copulatory apparatus. Scale bar: $\mathrm{A}=90 \mu \mathrm{m} ; \mathrm{B}=40 \mu \mathrm{m} ; \mathrm{C}$ and $\mathrm{D}=55 \mu \mathrm{m}$. 


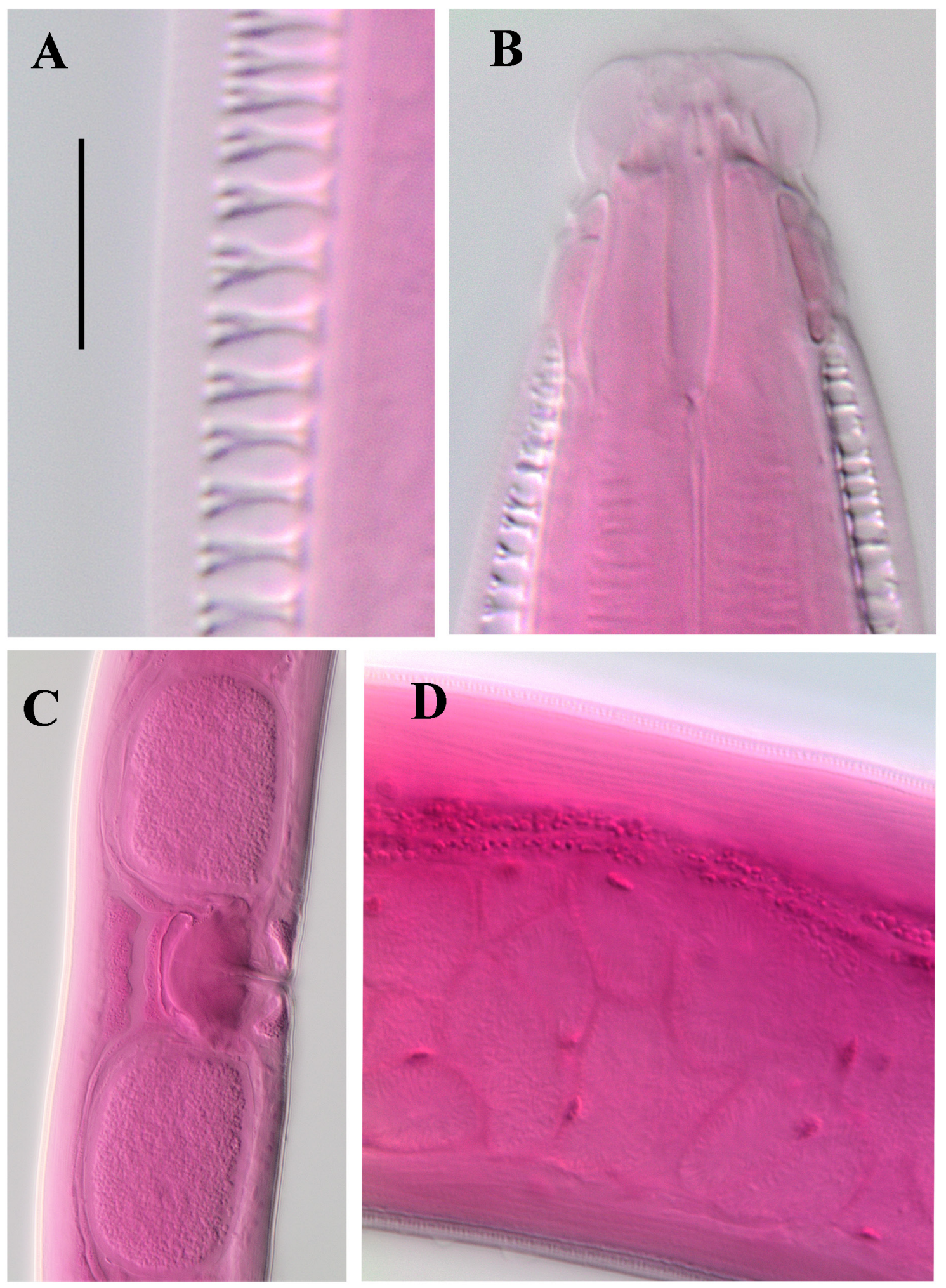

Fig. 11. Dorylaimopsis nodderi sp. nov. light micrographs. A. $q$ cuticle in pharyngeal region showing the cortical, median, and basal layers. B. Ventral view of $q$ head showing posterior portion of buccal cavity. C. + mid-body region showing vulva and mature eggs. D. Sperm cells with nuclei. Scale bar: $\mathrm{A}=8 \mu \mathrm{m} ; \mathrm{B}=12 \mu \mathrm{m} ; \mathrm{C}=50 \mu \mathrm{m} ; \mathrm{D}=30 \mu \mathrm{m}$. 
Table 4. Morphometrics $(\mu \mathrm{m})$ of Dorylaimopsis nodderi sp. nov.

\begin{tabular}{|c|c|c|c|}
\hline \multirow[t]{2}{*}{ Species } & \multicolumn{3}{|c|}{ Dorylaimopsis nodderi sp. nov. } \\
\hline & Male Holotype & Male Paratypes & Female Paratypes \\
\hline $\mathbf{n}$ & & 4 & 4 \\
\hline $\mathbf{L}$ & 1524 & $1199-1602$ & $1080-1656$ \\
\hline a & 23 & $18-27$ & $20-26$ \\
\hline b & 7 & $6-7$ & $6-7$ \\
\hline c & 14 & $11-13$ & $11-13$ \\
\hline Head diam. & 14 & $12-14$ & 13-19 \\
\hline Mouth diam. & 3 & $1-2$ & $2-4$ \\
\hline Length of cephalic setae & 6 & $4-7$ & $5-7$ \\
\hline Amphid height & 11 & $9-11$ & $7-9$ \\
\hline Amphid width & 10 & 10 & $8-9$ \\
\hline Amphid width/cbd (\%) & 59 & $63-67$ & $40-53$ \\
\hline Amphid from anterior end & 8 & $7-8$ & $7-9$ \\
\hline Nerve ring from anterior end & 106 & $100-125$ & $93-132$ \\
\hline Nerve ring cbd & 47 & $40-55$ & $41-57$ \\
\hline Excretory pore from anterior end & 134 & $115-148$ & $105-153$ \\
\hline Pharynx length & 224 & $196-250$ & $185-264$ \\
\hline Pharynx cbd & 65 & $45-75$ & $50-74$ \\
\hline Pharyngeal bulb diam. & 34 & $29-50$ & $34-52$ \\
\hline Max. body diam. & 65 & $44-87$ & $50-81$ \\
\hline Spicule length & 112 & $98-109$ & - \\
\hline Gubernacular apophyses length & 40 & $31-39$ & - \\
\hline Anal body diam. & 44 & $38-58$ & $37-54$ \\
\hline Tail length & 112 & $109-128$ & $98-134$ \\
\hline Tail length/abd & 2.5 & $2.2-2.9$ & $2.5-3.0$ \\
\hline $\mathbf{V}$ & - & - & $497-790$ \\
\hline$\% \mathrm{~V}$ & - & - & $46-48$ \\
\hline Vulval body diam. & - & - & $49-81$ \\
\hline
\end{tabular}

\section{Diagnosis and relationships}

Dorylaimopsis nodderi sp. nov. is characterised by cuticle with lateral differentiation consisting of three longitudinal rows of larger dots in pharyngeal and caudal regions, two rows of larger dots in middle region of body, cephalic setae 4-7 $\mu \mathrm{m}$ long, amphideal fovea with 2.5-3.0 turns, and spicules 98-112 $\mu \mathrm{m}$ long with rounded ventral projection at one third of spicule length from distal end, giving appearance of a joint.

Dorylaimopsis nodderi sp. nov. differs from most other species of the genus (except D. punctata Ditlevsen, 1918 and D. magellanense Chen \& Vincx, 1998) in the presence of spicules with ventral projections. D. nodderi sp. nov. can be distinguished from $D$. punctata by its smaller body length (1080$1656 \mathrm{vs} .>1900 \mu \mathrm{m}$ ), presence of three rows of enlarged dots in pharyngeal and caudal regions of both males and females (two rows in D. punctata), and fewer pre-cloacal supplements (12-16 vs. 18-22). D. nodderi sp. nov. can be distinguished from D. magellanense by its smaller body length (1199-1602 vs. $1708-2261 \mu \mathrm{m}$ ), and the smaller number of rows of enlarged dots in the lateral fields: three rows in pharyngeal and caudal regions (four or five in D. magellanense) and two rows in middle region of body (three or four in D. magellanense). 


\section{Dichotomous key to all known species of Dorylaimopsis}

(modified from Jensen (1979) and Zhang (1992))

1. Cuticle without lateral longitudinal rows of coarse dots along entire body .................................... 2

- Cuticle with longitudinal rows of dots along entire length of body ................................................ 4

2. Lateral longitudinal rows of coarse dots present posterior to pharyngeal region only....................... 3

- Lateral longitudinal rows of coarse dots completely absent

Dorylaimopsis metatypica Chitwood, 1936

3. Lateral differentiation of cuticle consisting of 1-3 longitudinal rows of dots beginning posterior to pharynx, gubernaculum with swollen distal end

D. coomansi Muthumbi et al., 1997

- Lateral differentiation of cuticle consisting of 3-5 longitudinal rows of dots beginning posterior to pharynx, triangular gubernaculum tapering distally.....

D. turneri Zhang, 1992

4. Cuticle laterally with two longitudinal rows of coarse dots in middle body region of both sexes...... 5

- Cuticle laterally with more than two longitudinal rows of coarse dots in at least one of the sexes .. 14

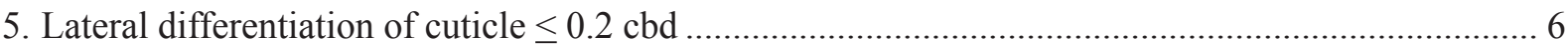

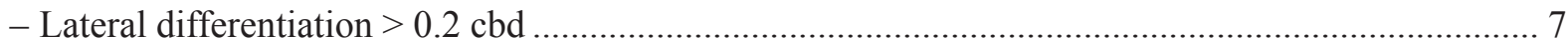

6. Body length 2.5-3.2 mm; spicules with ventral projection

D. punctata Ditlevsen, 1918

- Body length 1.3-1.9 mm; spicules without ventral projection D. rabalaisi Zhang, 1992

7. Spicules jointed, with ventral projection, or simple 8

- Spicules jointed and irregularly cuticularised

D. mediterranea Grimaldi-de Zio, 1968

8. Spicules jointed, with two equal segments

D. perfecta (Cobb, 1920)

- Spicules otherwise

9. Spicules without distal hooks or projections

- Spicules with distal hooks or ventral projections

10. Gubernaculum with irregular base; gubernacular apophyses with blunt distal end

D. nini (Inglis, 1961)

- Gubernaculum with smooth base; gubernacular apophyses with pointed or rounded distal end .......11

11. Gubernaculum with pointed end

D. peculiaris Platonova, 1971

- Gubernaculum with rounded end

12. Male body length $<2100 \mu \mathrm{m}$, two equal testes, spicules with poorly developed capitulum, spicule length 1.7-1.9 abd

D. gerardi Muthumbi et al., 1997

- Male body length $>2100 \mu \mathrm{m}$, anterior testis larger than posterior testis and with larger sperm, spicules with well-developed capitulum, spicule length 1.8-2.4 abd

D. variabilis Muthumbi et al., 1997

13. Spicules with small distal hook; gubernaculum with lateral projections ... D. pellucida (Cobb, 1920)

- Spicules with rounded ventral projection at one third of spicule length from distal end; cuticle laterally with three longitudinal rows in pharyngeal and caudal regions, two rows in middle region

D. nodderi sp. nov.

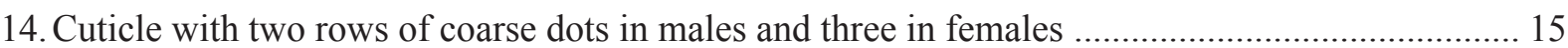

- Cuticle with more than two rows of coarse dots in males .............................................................. 16 
15. Spicules distally acute

D. poriferum (Cobb, 1920)

- Spicules distally with a subterminal hook

D. timmi Jensen, 1979

16. Cuticle with three rows of coarse dots in middle body region of males

- Cuticle with four or five rows of coarse dots in middle body region of males

17. Cuticle with 3 rows of coarse dots along entire length of body of both males and females

D. halongensis Tu et al., 2008

- Cuticle with three rows of coarse dots in middle region of body, four to eight rows elsewhere (males and females)

D. janetae (Inglis, 1963)

18. Spicules without subterminal hook

D. magellanense Chen and Vincx, 1998

- Spicule with subterminal hook; spicule length 3 abd

D. angelae (Inglis, 1967)

Genus Hopperia Vitiello, 1969

\section{Diagnosis (modified from Jensen (1979))}

Dorylaimopsinae. Cuticle with lateral differentiation of irregularly-spaced larger dots, sometimes arranged in transverse rows; posterior portion of buccal cavity cylindrical, with three thorn-like teeth at border to anterior portion (except in H. hexadentata Hope \& Zhang, 1995 which has six teeth); ovaries usually outstretched, reflexed ovaries sometimes present; spicules arcuate; gubernaculum with caudal or dorso-caudal apophyses.

\section{Type species}

Hopperia massiliensis Vitiello, 1969

\section{Hopperia ancora sp. nov. urn:1sid:zoobank.org:act:42970B24-CF4B-4D54-9CFF-B278BE09C60C}

Figs 12-14, Table 5

\section{Etymology}

The species name is derived from the Latin ancora (= anchor), in reference to the distal hook on the spicules.

\section{Material examined}

\section{Holotype}

$\mathrm{O}^{\AA}$, collected 7 Jun. 2007 , Challenger Plateau (264 m water depth), $39.6463^{\circ} \mathrm{S}, 172.1530^{\circ} \mathrm{E}$. Characteristics of surface sediment layer $(0-5 \mathrm{~mm})$ : mostly silt/clay $(90.1 \%)$, with very fine sand $(3.0 \%)$, and fine sand (3.0\%); calcium carbonate content: $27.4 \%$; total organic matter content: $1.94 \%$; chloroplastic pigment concentration: $3039 \mathrm{ng} / \mathrm{gDW}_{\text {sediment }}$ (NIC 84448).

\section{Paratypes}

3 ऊิ $\widehat{\partial}$ and 3 우, same data as holotype (NIC 84449).

\section{Description}

\section{Males}

Body cylindrical, tapering slightly towards anterior extremity. Cuticle punctated from level of amphid to near tail tip, with lateral differentiation consisting of larger, irregularly-spaced dots. Eight longitudinal rows of short, sparse somatic setae. Head set-off by depression at level of cephalic setae. Six inner labial papillae, six outer labial papillae, and four conical cephalic setae in three distinct circles. Amphideal 


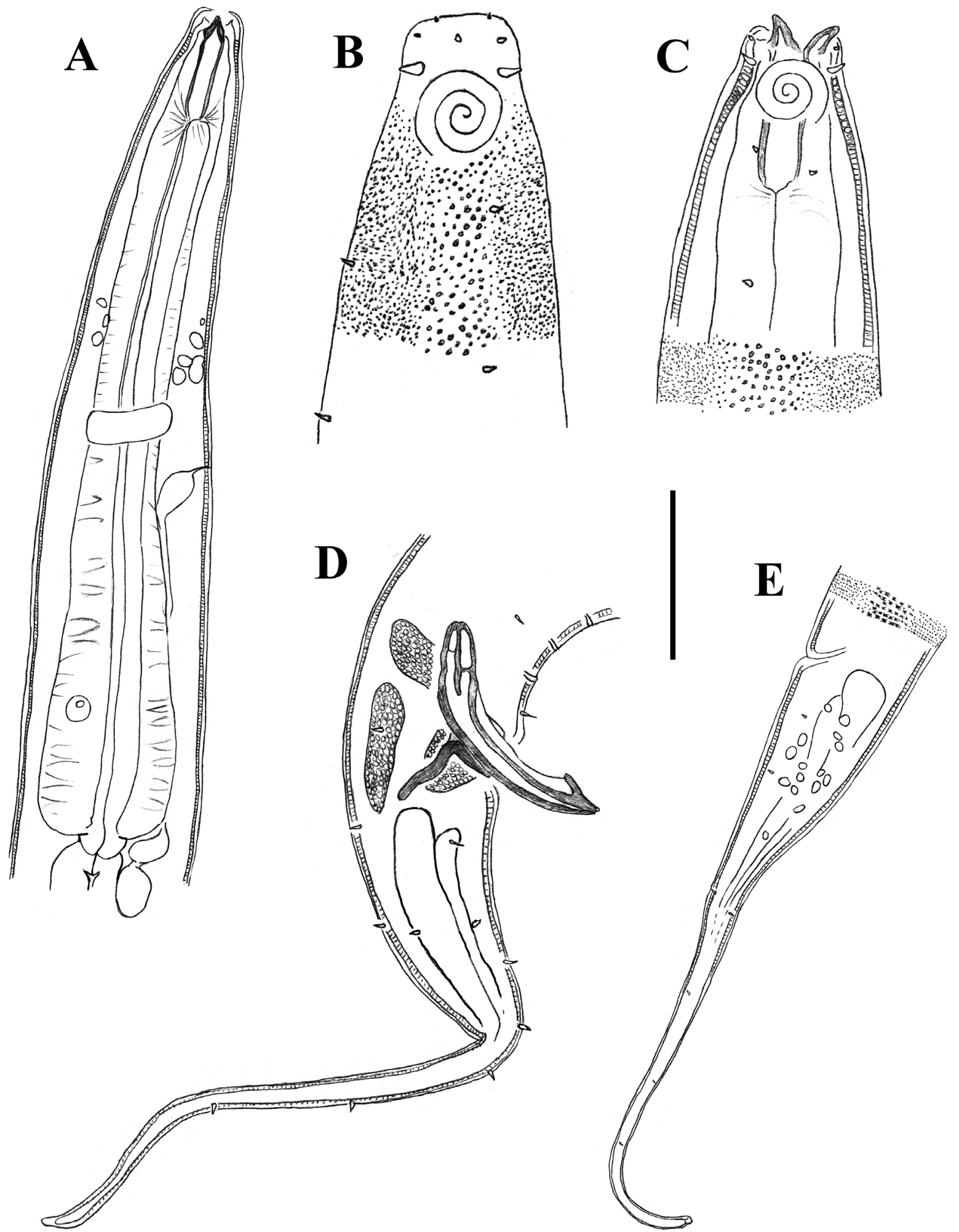

Fig. 12. Hopperia ancora sp. nov. A. Anterior region of $\widehat{\partial}$. B. Lateral surface view of $\widehat{\partial}$ head. C. Lateral view of $q$ head. D. $\delta$ posterior body region, showing copulatory apparatus. E. $q$ tail. Scale bar: $\mathrm{A}=45 \mu \mathrm{m} ; \mathrm{B}$ and $\mathrm{C}=20 \mu \mathrm{m} ; \mathrm{D}=35 \mu \mathrm{m} ; \mathrm{E}=50 \mu \mathrm{m}$. 
A

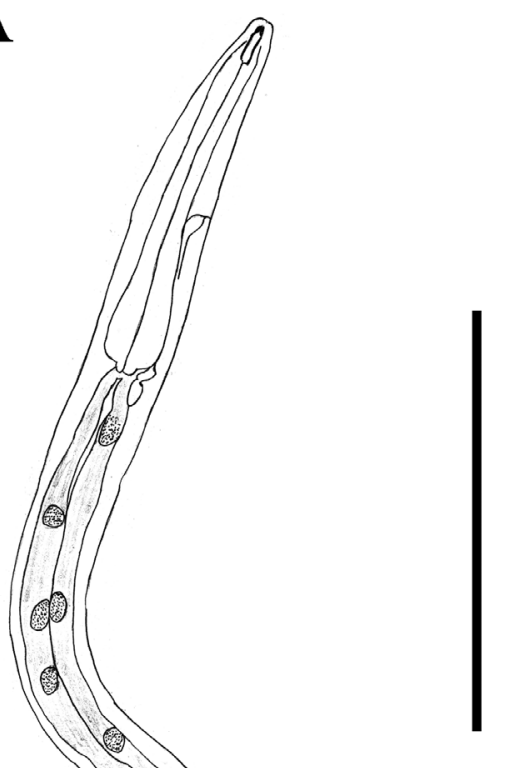

B
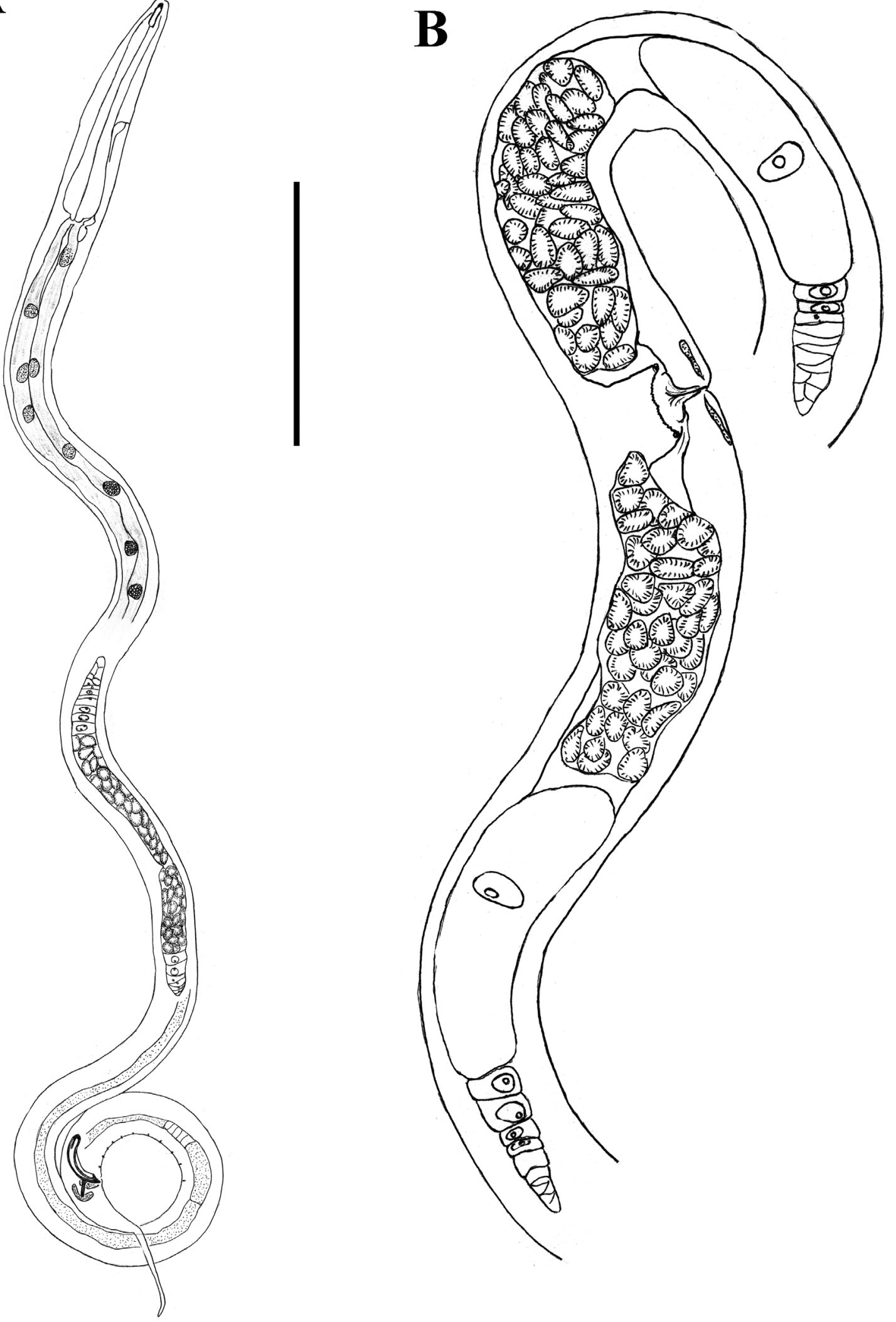

Fig. 13. Hopperia ancora sp. nov. A. Entire $\partial^{\lambda}$. B. $q$ reproductive system. Scale bar: $A=240 \mu \mathrm{m}$; $\mathrm{B}=110 \mu \mathrm{m}$. 
fovea spiral, 2.5-3.0 turns, located immediately posterior to cephalic setae.Anterior portion of buccal cavity cup-shaped. Posterior portion of buccal cavity cylindrical, strongly cuticularised, 4-5 $\mu \mathrm{m}$ wide, 20-27 $\mu \mathrm{m}$ deep, with three strongly cuticularised teeth at border to anterior portion. Teeth everted in some specimens (Fig. 14A). Marginal tubes of pharynx beginning from base of stoma. Pharynx gradually swelling, not forming true bulb. Cardia short. Nerve ring near middle of pharynx, situated anteriorly to
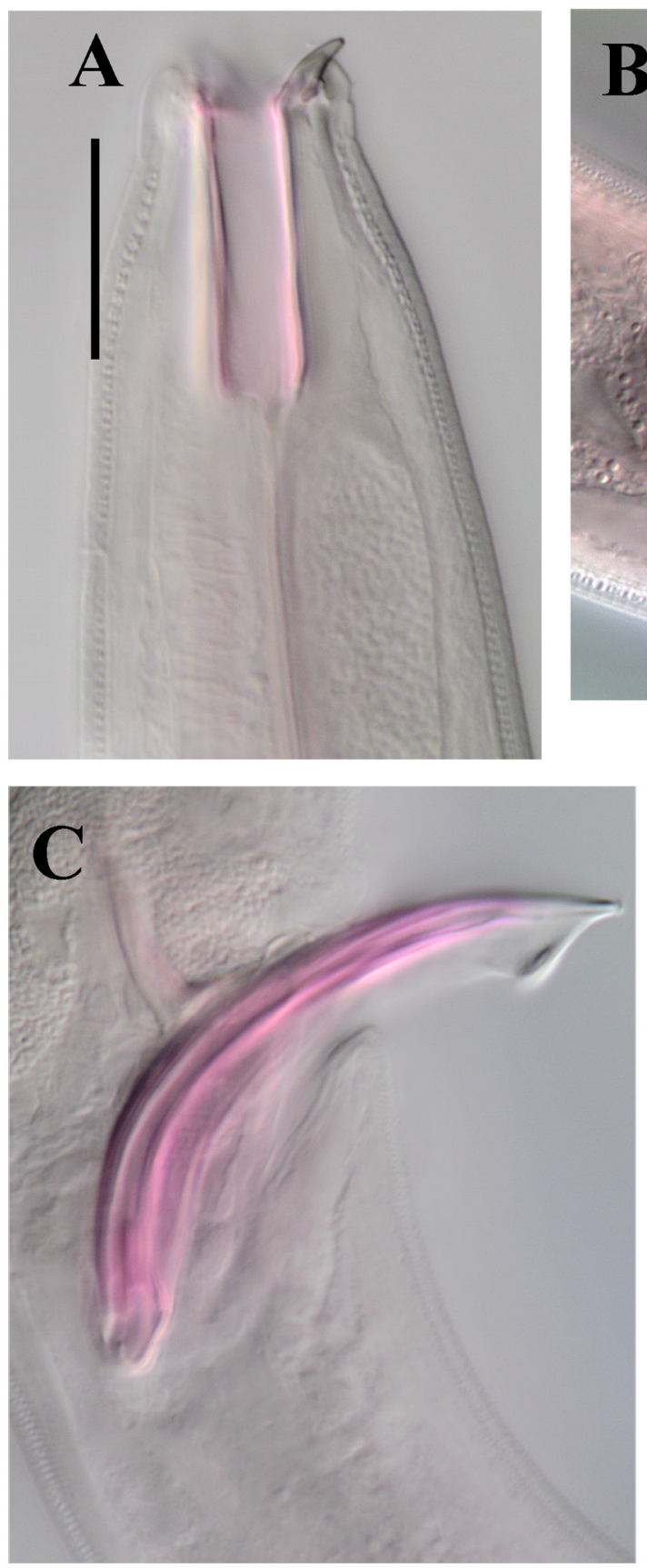
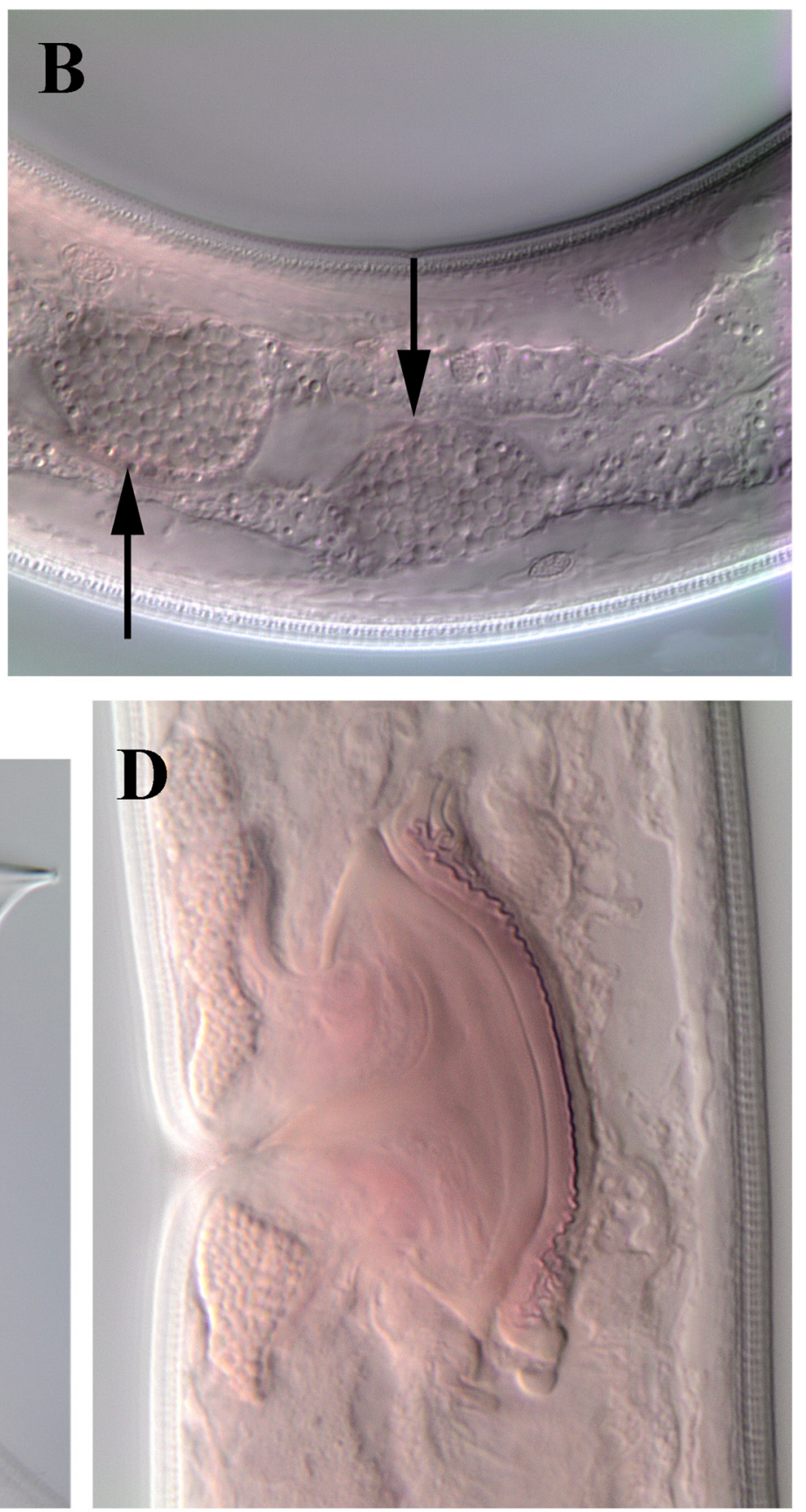

Fig. 14. Hopperia ancora sp. nov. light micrographs. A. $\delta^{\lambda}$ head, showing everted tooth. B. Intestine wall of $\lambda$, with arrows pointing to oval cells with larger, more closely packed granules than surrounding cells. C. Everted spicule. D. Vulva. Scale bar: A, D $=15 \mu \mathrm{m} ; \mathrm{B}=22 \mu \mathrm{m} ; \mathrm{C}=20 \mu \mathrm{m}$. 
Table 5. Morphometrics ( $\mu \mathrm{m})$ of Hopperia ancora sp. nov. and H. beaglense Chen and Vincx, 1998.

\begin{tabular}{|c|c|c|c|c|c|}
\hline \multirow[t]{3}{*}{ Species } & \multicolumn{3}{|c|}{ Hopperia ancora sp. nov. } & \multicolumn{2}{|c|}{ Hopperia beaglense Chen \& Vincx, 1998} \\
\hline & \multicolumn{2}{|c|}{ Males } & \multirow{2}{*}{$\begin{array}{c}\text { Females } \\
\text { Paratypes }\end{array}$} & \multirow[b]{2}{*}{ Males } & \multirow[b]{2}{*}{ Females } \\
\hline & Holotype & Paratypes & & & \\
\hline n & & 3 & 3 & 5 & 4 \\
\hline $\mathbf{L}$ & 2011 & $1876-1986$ & $1985-2139$ & $1446-1616$ & $1573-1856$ \\
\hline $\mathbf{a}$ & 47 & $32-46$ & $29-50$ & $31-36$ & $29-36$ \\
\hline b & 9 & $8-9$ & $8-9$ & $7-8$ & 8 \\
\hline c & 12 & $10-11$ & 10 & $14-17$ & $16-19$ \\
\hline Head diam. & 16 & $15-17$ & $15-17$ & $13-14$ & $14-15$ \\
\hline Mouth diam. & 4 & 5 & $4-5$ & $3-6$ & $3-5$ \\
\hline Length of cephalic setae & 3 & $2-3$ & 3 & $4-6$ & $4-6$ \\
\hline Amphid height & 11 & 11 & $10-11$ & $10-11$ & 8 \\
\hline Amphid width & 12 & $11-12$ & $9-12$ & $10-12$ & $9-10$ \\
\hline Amphid width/cbd (\%) & 58 & $58-64$ & $50-60$ & $63-71$ & $53-59$ \\
\hline Amphid from anterior end & 7 & $5-7$ & 5 & 6 & 6 \\
\hline Nerve ring from anterior end & 90 & $82-104$ & $97-111$ & $82-103$ & $98-113$ \\
\hline Nerve ring cbd & 45 & $38-44$ & $41-49$ & $34-38$ & $31-45$ \\
\hline Excretory pore from anterior end & 118 & $116-124$ & $123-137$ & $115-124$ & $133-140$ \\
\hline Pharynx length & 218 & $210-236$ & $233-240$ & $187-208$ & $208-235$ \\
\hline Pharynx cbd & 53 & $48-62$ & $48-62$ & $39-44$ & $48-60$ \\
\hline Pharyngeal bulb diam. & 37 & $35-47$ & $33-47$ & $27-30$ & $37-42$ \\
\hline Max. body diam. & 43 & $41-62$ & $40-69$ & $42-48$ & $49-64$ \\
\hline Spicule length & 62 & $54-68$ & - & $48-58$ & - \\
\hline Gubernacular apophyses length & 18 & $19-21$ & - & $25-31$ & - \\
\hline Anal body diam. & 38 & $36-44$ & $32-36$ & $35-40$ & $38-45$ \\
\hline Tail length & 167 & $175-206$ & $202-212$ & $92-100$ & $100-105$ \\
\hline Tail length/abd & 4.4 & $4.7-4.9$ & $5.9-6.3$ & $2.3-2.9$ & $2.2-2.7$ \\
\hline $\mathbf{V}$ & - & - & 934-1041 & - & $785-953$ \\
\hline$\% \mathrm{~V}$ & - & - & $47-49$ & - & $50-52$ \\
\hline Vulval body diam. & - & - & $40-67$ & - & $48-61$ \\
\hline
\end{tabular}

excretory pore. Cellular body of ventral gland situated just posteriorly to cardia. Intestine wall with numerous small, clear granules, and oval cells with more closely-packed granules (Figs 13A, 14B).

Reproductive system diorchic, opposed, outstretched. Anterior testis to left of intestine and posterior testis to right of intestine in all specimens except one specimen with opposite arrangement. Sperm cells globular with outer radial lines. Spicules paired, equal, arcuate, 1.5-1.7 abd long, with swollen proximal end and central internal cuticularised projection (lamella) extending one fourth of spicule length from proximal end. Spicules with velum and conspicuous hook-like projection at distal end (Fig. 14C). Gubernaculum appearing to completely surround spicules, with bent dorso-caudal apophyses. Rectal gland surround distal end of spicules and gubernaculum. Eleven to thirteen tubular pre-cloacal supplements, one pre-cloacal seta. Tail long, conico-cylindrical, gradually tapering, with several short caudal setae and no terminal setae. Three caudal glands. 


\section{Females}

Similar to males, but with slightly longer tail. Reproductive system didelphic, opposed, outstretched, with anterior and posterior branches either to left or right of intestine, but always on opposite sides. Vulva slightly pre median. Granular vaginal glands present, pars proximalis vaginae surrounded by constrictor muscle (Fig. 14D).

\section{Diagnosis and relationships}

Hopperia ancora sp. nov. is characterised by body length $1876-2011 \mu \mathrm{m}$, short conical cephalic setae, amphideal fovea with 2.5-3.0 turns, buccal cavity with three teeth, spicules 54-68 $\mu \mathrm{m}$ in length with hook-like projection at distal end, gubernaculum with bent apophyses, 11-13 pre-cloacal supplements, and gradually tapering conico-cylindrical tail without swollen tip or terminal setae.

Hopperia ancora sp. nov. most closely resembles H. massiliensis Vitiello, 1969 and H. indiana Muthumbi et al., 1997 in body size, values of $a, b$, and $c$, size and shape of the cephalic setae, amphideal fovea, and tail, but can be distinguished from them (and all other species of the genus) by the presence of a conspicuous hook-like projection at distal end of spicules.

Hopperia beaglense Chen \& Vincx, 1998

Figs 15-17, Table 5

\section{Material examined}

$5 \widehat{\partial} \delta^{\lambda}$ and 4 q 9 , collected 5 May 2010, Kaikoura Canyon axis (1061 m water depth), $42.5081^{\circ} \mathrm{S}$, $173.6325^{\circ} \mathrm{E}$ (NIC 84450).

\section{Description}

\section{Males}

Body cylindrical, tapering slightly towards anterior extremity. Cuticle punctate from level of amphid to near tail tip, with lateral differentiation consisting of larger, irregularly-spaced dots. Somatic setae short and sparse, in four dorso- and ventro-lateral longitudinal rows. Six inner labial papillae, six outer labial papillae, and four cephalic setae in three distinct circles. Anterior portion of buccal cavity cupshaped. Posterior portion of buccal cavity cylindrical, strongly cuticularised, 24-26 $\mu \mathrm{m}$ deep, extending into three strongly cuticularised teeth at border to anterior portion. Teeth everted in some specimens. Cuticularisation of posterior portion of buccal cavity extends posterior to junction of marginal tubes with pharyngeal lumen (Fig. 15B). Amphideal fovea spiral, 3.0-3.5 turns, located immediately posterior to cephalic setae. Pharynx with oval posterior bulb. Cardia short. Nerve ring near middle of pharynx, situated anteriorly to secretory-excretory pore. Cellular body of ventral gland large (up to 37 x $20 \mu \mathrm{m}$ ), situated just posteriorly to cardia. Intestine wall with numerous small, clear granules, and small clusters of round, clear inclusions, sometimes with smaller orange-brown granules (Fig. 17B).

Reproductive system diorchic, opposed, outstretched. Anterior testis to left of intestine and posterior testis to right of intestine. Spicules paired, equal, arcuate, strongly cuticularised, 1.3-1.6 abd long, with swollen proximal end and internal cuticularise projection (lamella) extending one fourth of spicule length from proximal end, no velum. Gubernaculum with straight dorso-caudal apophyses, tapering distally. One pre-cloacal seta and 7-8 conspicuous, cup-shaped pre-cloacal supplements. Tail short, cylindrical, with rounded tip, several short caudal setae and no terminal setae. Three large caudal glands and well-developed spinneret (Fig. 17C).

\section{Females}

Similar to males, but slightly greater maximum body diameter and smaller amphids, 3.0 turns. Reproductive system didelphic, opposed, outstretched, with anterior branch to left of intestine and 


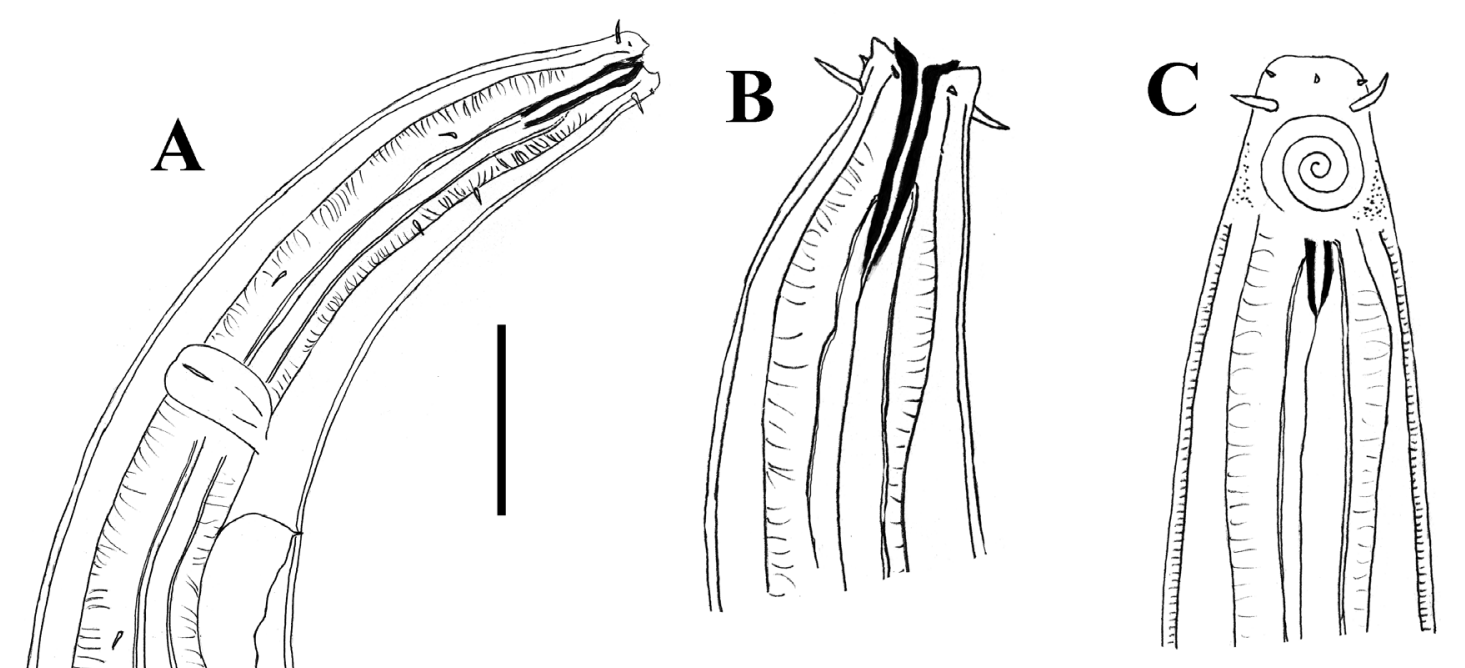


posterior branch to right of intestine, except for one individual with opposite arrangement. Vulva slightly post median. Granular vaginal glands present, vagina uterina surrounded by constrictor muscle.

\section{Discussion}

My specimens closely resemble the description given by Chen \& Vincx (1998) based on specimens from the Beagle Channel, Chile (100-110 m water depth). My specimens, however, have slightly lower $a$

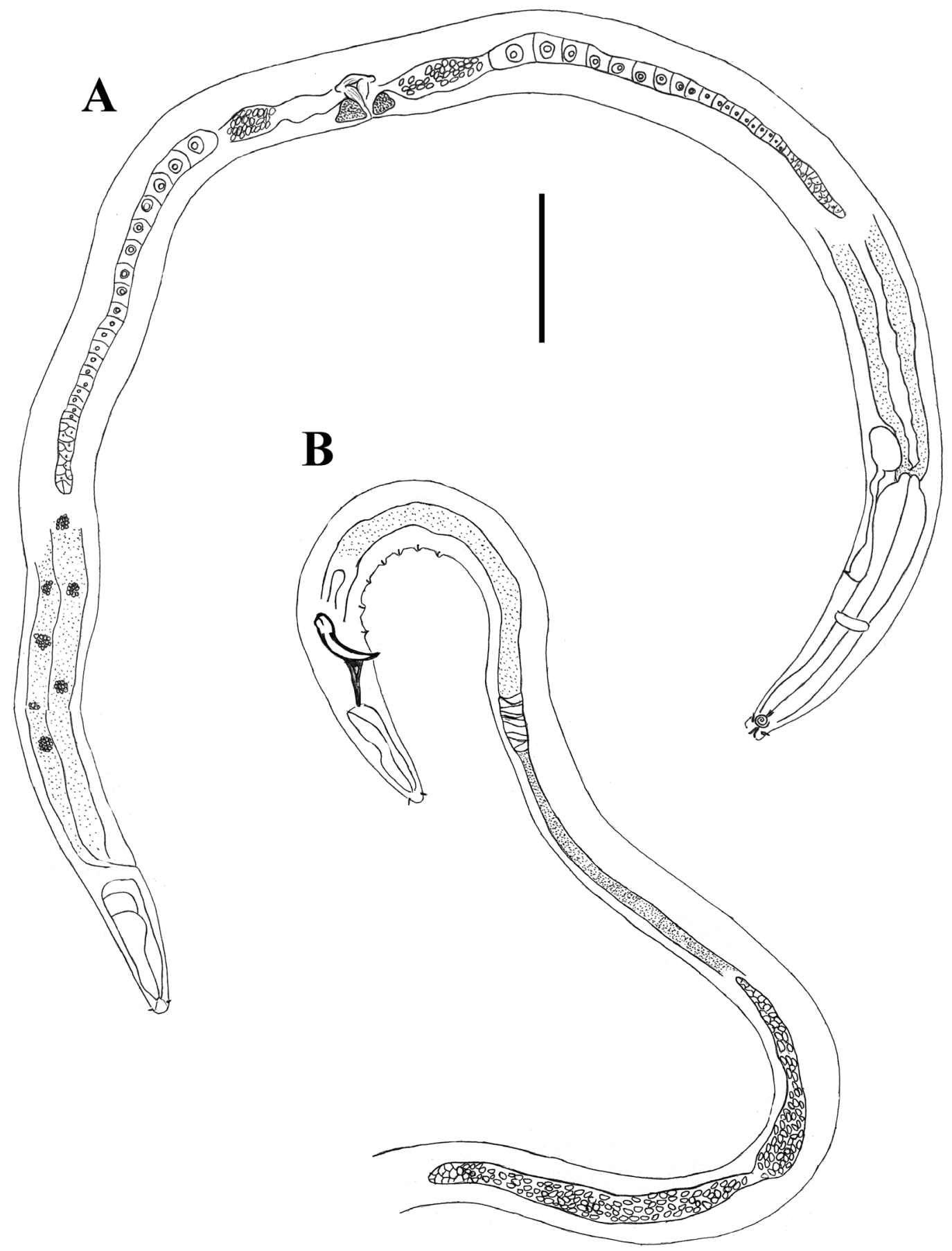

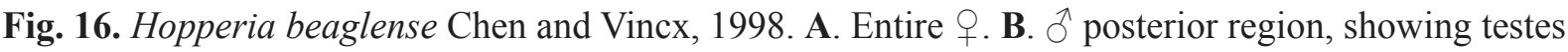
and copulatory apparatus. Scale $\mathrm{bar}=100 \mu \mathrm{m}$. 
(range: $29-36$ vs. $30-41$; mean: 34.2 vs. 39.1 ) and $c$ values (range: $14-19$ vs. 19-22; mean: 15.8 vs. 20.6), have 7-8 supplements instead of 6-7, and lack the supplement-like structure situated halfway down the ventral side of the tail (Fig. 15F). This is the first record of this species outside the type locality.

Within the Dorylaimopsinae, cuticularisation of the posterior buccal cavity does not usually extend into the pharyngeal lumen (i.e., cuticularisation stops before marginal tubes begin) (fig. 2E in Jensen 1979). My specimens are unusual in that the cuticularisation of the buccal cavity extends into the anteriormost portion of the pharyngeal lumen (see Fig. 15B). It is unclear whether this is also a feature of the type specimens, however, because Chen \& Vincx (1998) did not describe the marginal tubes.
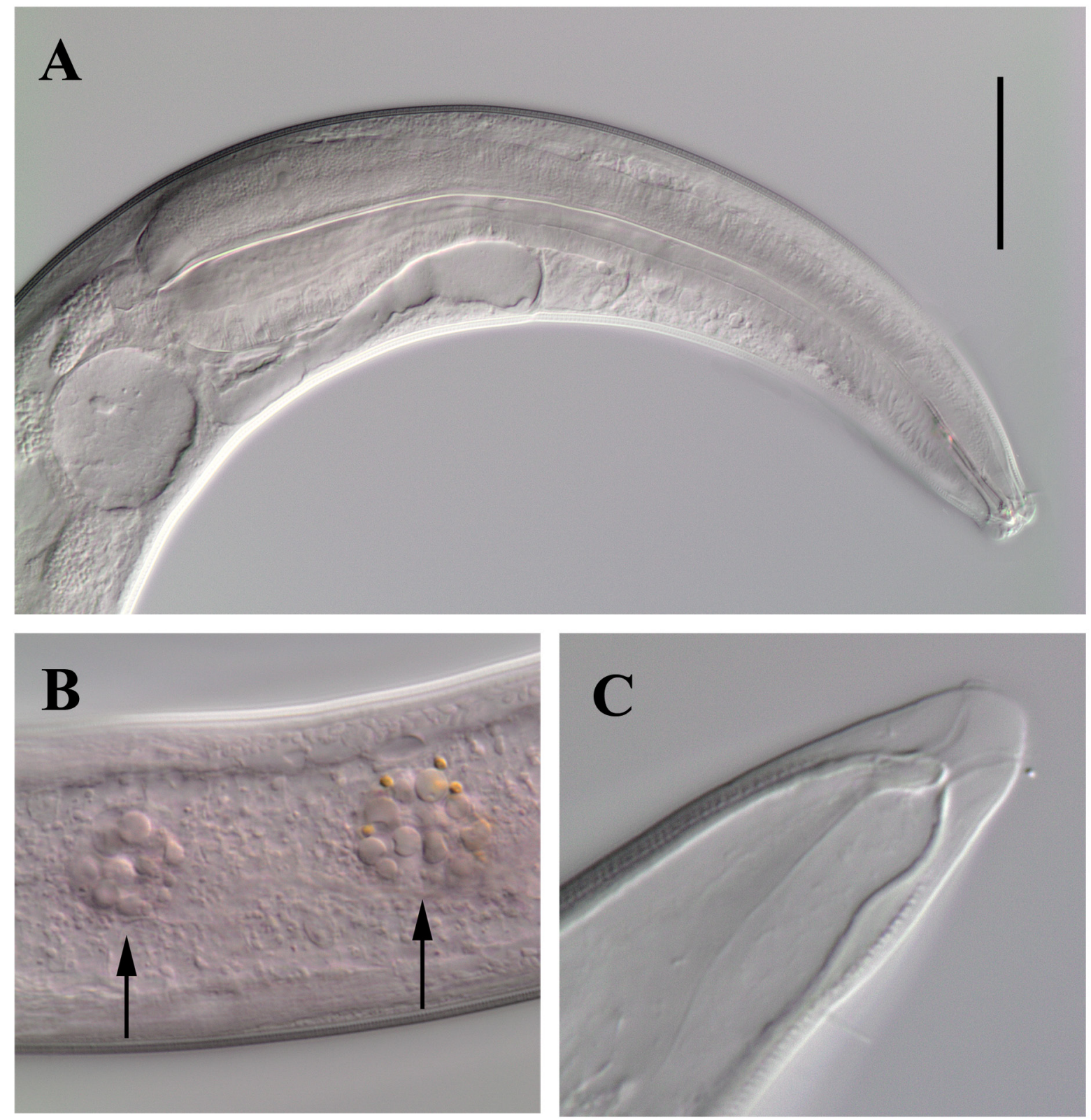

Fig. 17. Hopperia beaglense Chen and Vincx, 1998 light micrographs. A. Anterior region of $\widehat{\partial}$. B. $\widehat{\partial}$ gut, showing clusters of small round inclusions with smaller orange-brown granules. C. Spinneret. Scale bar: $\mathrm{A}=30 \mu \mathrm{m} ; \mathrm{B}=12 \mu \mathrm{m} ; \mathrm{C}=24 \mu \mathrm{m}$. 
Key to all known species of Hopperia

(modified from Pastor de Ward (2004))

1. Cuticle with irregularly-distributed dots in the lateral fields

2. Cuticle with four longitudinal rows of coarse dots in lateral fields in anterior half or entire body length

Hopperia communis Gagarin \& Thanh, 2006

3. Stoma armed with three teeth at juncture of anterior and posterior portions of buccal cavity ............ 4

- Stoma armed with six teeth at juncture of anterior and posterior portions of buccal cavity. H. hexadentata Hope and Zhang, 1995

4. Spicules without joint 5

- Spicules jointed; cephalic setae 0.4 hd; > 13 small pre-cloacal supplements; tail conico-cylindrical with swollen tip and terminal setae

H. metatypica (Chitwood, 1936)

5. Spicules without distal hook. 6

- Spicules with distal hook; short conical cephalic setae; tail without terminal setae

H. ancora sp. nov.

6. Spicules without sharp proximal bend pointing ventrally ...... 7

- Spicules with sharp proximal bend; tail conico-cylindrical with swollen tip and terminal setae H. mira Gagarin and Thanh, 2006

7. Tail without conspicuous spinneret

- Tail with conspicuous spinneret; terminal setae absent; cylindrical portion of tail $>50 \%$ tail length

H. dolichura Gagarin and Thanh, 2006

8. Length of posterior cylindrical portion of buccal cavity $>1.5$ amphid diameters

- Length of posterior cylindrical portion of buccal cavity $<1.5$ amphid diameters; 12 pre-cloacal supplements; spicules 0.5 abd

H. patagonica Pastor de Ward, 2004

9. Length of posterior cylindrical portion of buccal cavity $=1.5-2.5$ amphid diameters 10

- Length of posterior cylindrical portion of buccal cavity $>2.5$ amphid diameters

10. Spicules $\leq 2.0 \mathrm{abd}$

- Spicules > 2.0 abd; pre-cloacal supplements present; tail with terminal setae

H. muscatensis Warwick, 1973

11. > 10 pre-cloacal supplements; spicules $69 \mu \mathrm{m}$

H. indiana Muthumbi et al., 1997

- $<10$ pre-cloacal supplements; spicules 37-44 $\mu \mathrm{m}$ H. australis Jensen, 1992

12. Tail tip not swollen

- Tail tip swollen

13. Reflexed ovaries; 8 pre-cloacal supplements

H. dorylaimopsoides (Allgen, 1959)

- Outstretched ovaries; 6-8 pre-cloacal supplements; tail conical

H. beaglense Muthumbi et al., 1997

14. $<10$ pre-cloacal supplements; ovaries outstretched

$->10$ pre-cloacal supplements; ovaries reflexed

H. massiliensis Vitiello, 1969

15. Tail 3.5 abd; $\mathrm{a}=26-30$; spicules $1.4 \mathrm{abd}$

H. americana Pastor de Ward, 1984

- Tail 4.4 abd; a =31-40; spicules 1.8 abd H. arntzi Chen \& Vincx, 1998 
Genus Kenyanema Muthumbi et al., 1997

\section{Diagnosis (modified from Muthumbi et al. (1997))}

Dorylaimopsinae. Head region much narrower than the rest of the body; cuticle punctate and sometimes transverse striae are visible; inner and outer labial sensillae indistinct; four cephalic setae; tubular stoma without teeth; male reproductive system monorchic with outstretched anterior testis; females amphidelphic with outstretched ovaries; spicules arcuate; gubernaculum with caudal apophyses.

\section{Type species}

Kenyanema monorchis Muthumbi et al., 1997

Kenyanema monorchis Muthumbi et al., 1997

Figs 18-19, Table 6

\section{Material examined}

$3 \widehat{\partial} \delta$ and 3 Q $q$, collected 6 Apr. 2007 , southern flank of Chatham Rise (1240 m water depth), $44.4864^{\circ} \mathrm{S}$, $177.1407^{\circ} \mathrm{E}$. Characteristics of surface sediment layer $(0-5 \mathrm{~mm})$ : mostly silt/clay $(82.9 \%)$, with very fine sand $(10.2 \%)$, and fine sand $(8.4 \%)$; calcium carbonate content: $38.4 \%$; total organic matter content: $1.9 \%$; chloroplastic pigment concentration: $3501 \mathrm{ng} / \mathrm{gDW}_{\text {sediment }}(\mathrm{NIC} 84451)$.

\section{Description}

\section{Males}

Body cylindrical, tapering towards anterior extremity. Cuticle with transverse rows of dots, lateral differentiation consisting of larger, irregularly-spaced dots. Eight rows of short, sparse somatic setae. Head set-off by constriction immediately posterior to cephalic setae. Inner and outer labial sensillae indistinct, four cephalic setae. Amphideal fovea spiral, 2.5 turns, located at level of cephalic setae. Buccal cavity tubular, $4 \mu \mathrm{m}$ deep, $1 \mu \mathrm{m}$ wide, surrounded by pharyngeal muscles. Marginal tubes beginning from base of stoma. Pharynx gradually swelling, not forming true bulb, cardia short. Nerve ring near middle of pharynx, situated anteriorly to secretory-excretory pore. Cellular body of ventral gland near level of cardia. Intestine wall with numerous small, colourless granules, and clusters of clear circular inclusions, mostly in posterior portion of intestine (Fig. 18E).

Reproductive system monorchic with outstretched anterior testis to left of intestine. Mature sperm cells large, oval-shaped, up to $30 \mu \mathrm{m}$ long and $13 \mu \mathrm{m}$ wide. Spicules paired, equal, arcuate, 1.6-1.8 abd long, with swollen proximal end and central internal cuticularisated projection (lamella) extending from proximal end over one fifth of spicule length. Gubernaculum with pair of straight caudal apophyses. Rectal gland surrounds posterior portion of spicules and gubernaculum. Two to three small tubular precloacal supplements, one pre-cloacal seta. Tail conico-cylindrical with swollen tip, several short caudal setae and three short terminal setae. Three caudal glands and spinneret.

\section{Females}

Similar to males, but with slightly greater maximum body diameter, amphideal fovea slightly smaller, 2.0-2.25 turns. Reproductive system didelphic, opposed, outstretched, with sperm distributed over uteri and with branches either to the right or left of intestine, but always on opposite sides. Vulva slightly post median.

\section{Discussion}

My specimens closely resemble the description of Kenyanema monorchis Muthumbi et al., 1997 based on specimens from the Indian Ocean (500-1112 m water depth). My specimens, however, have a smaller 
A

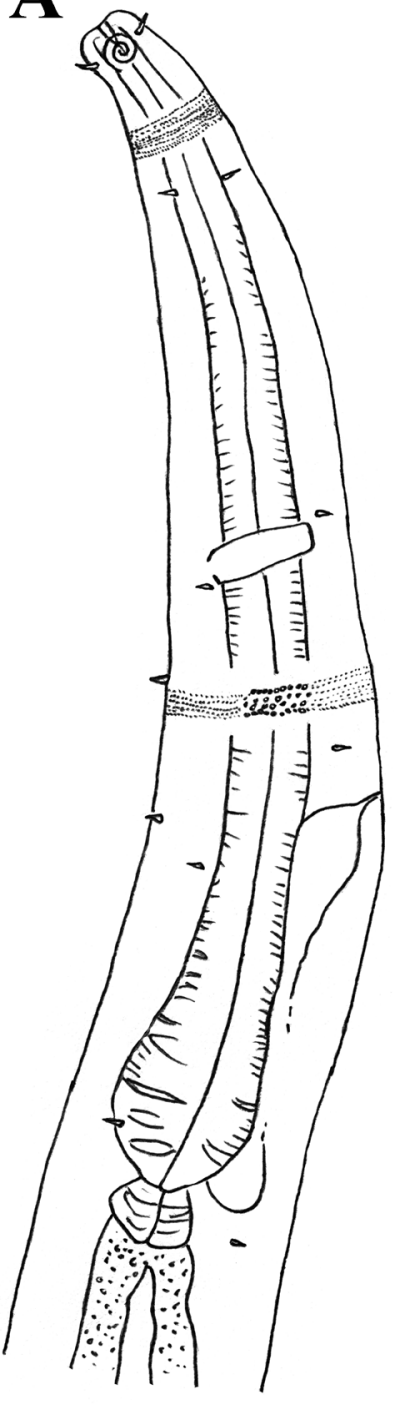

B

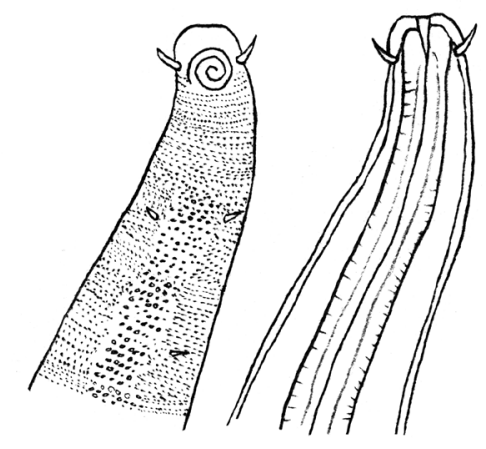

$\mathbf{E}$

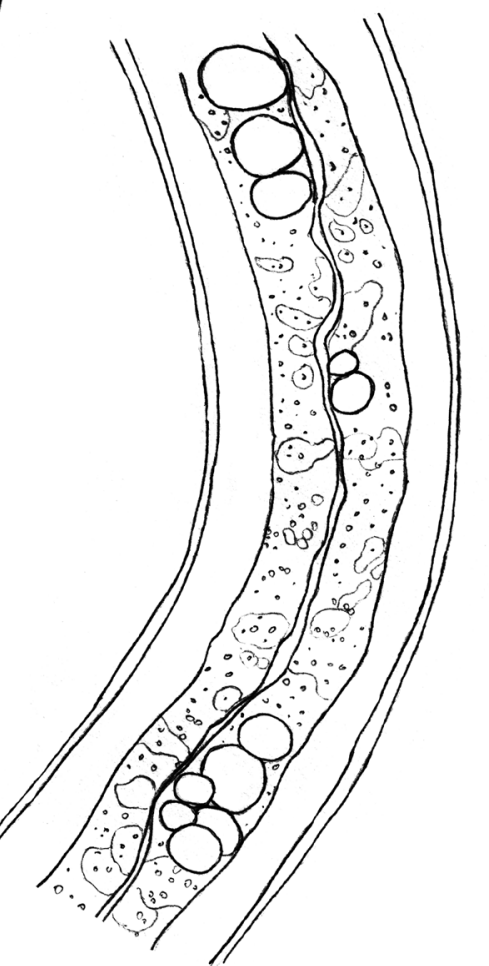

D
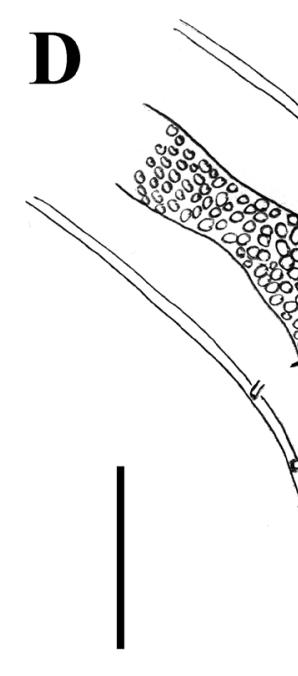
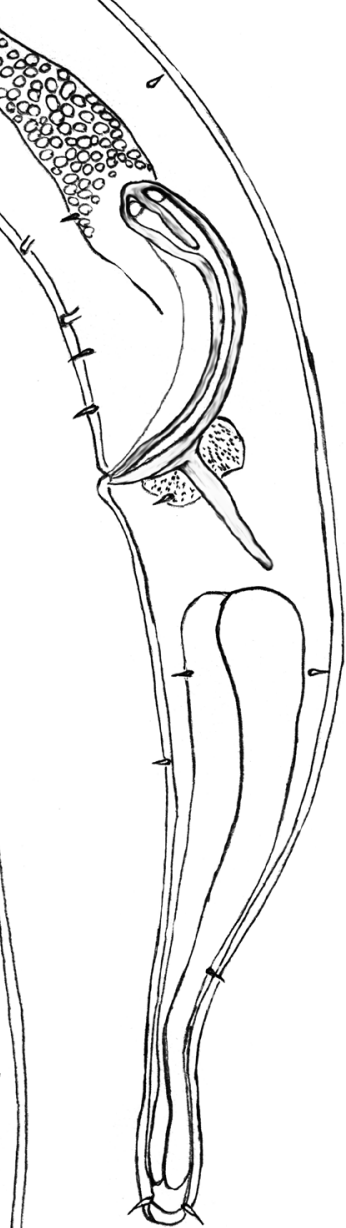

$\mathbf{F}$
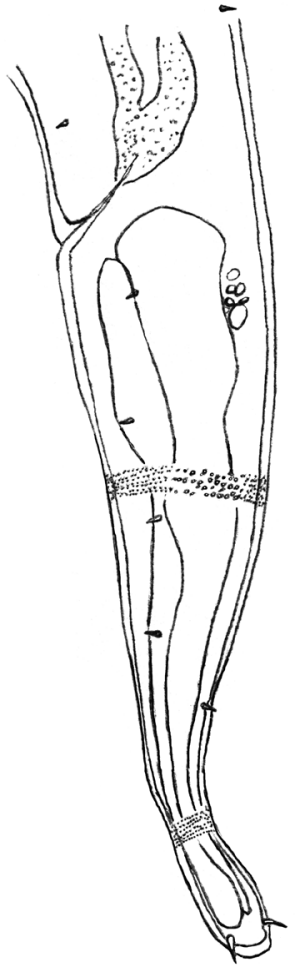

Fig. 18. Kenyanema monorchis Muthumbi et al., 1997. A. Anterior region of $q$; B: lateral surface view of $\hat{\partial}$ head. C. Lateral view of $\hat{\partial}$ head (cross-section). D. Posterior region of $\hat{\partial}$ showing copulatory apparatus. E. $q$ gut, posterior body region. F. $q$ tail. Scale bar $=25 \mu \mathrm{m}$. 

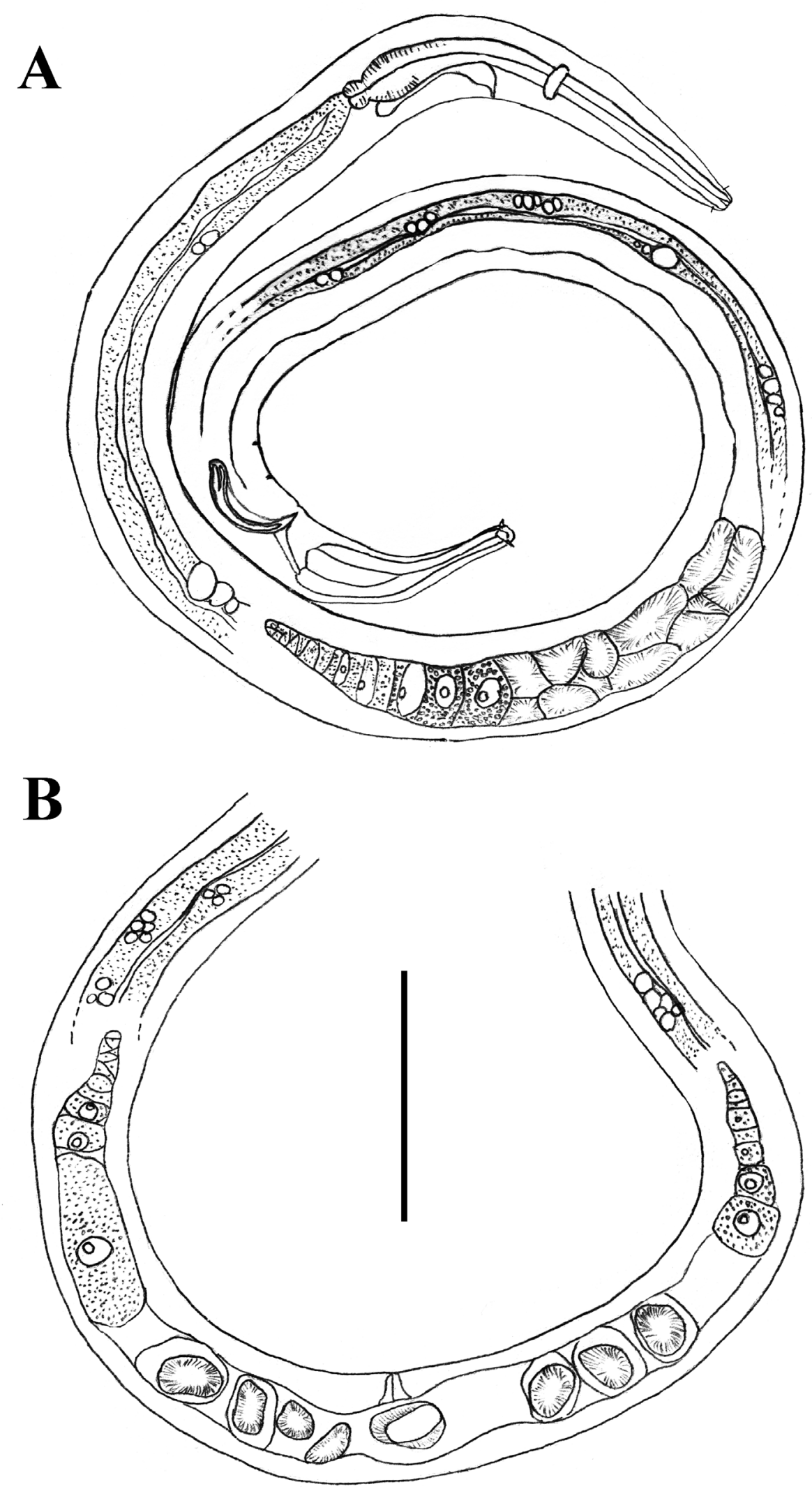

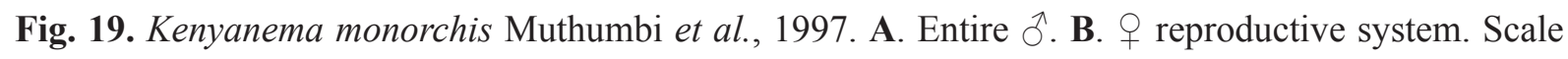
bar $=100 \mu \mathrm{m}$. 
Table 6. Morphometrics ( $\mu \mathrm{m})$ of Kenyanema monorchis Muthumbi et al., 1997.

\begin{tabular}{|c|c|c|}
\hline \multirow[t]{2}{*}{ Species } & \multicolumn{2}{|c|}{ Kenyanema monorchis Muthumbi et al., 1997} \\
\hline & Males & Females \\
\hline $\mathbf{n}$ & 4 & 3 \\
\hline $\mathbf{L}$ & $1020-1107$ & $1070-1205$ \\
\hline a & 29-39 & $27-29$ \\
\hline b & 7 & 8 \\
\hline c & $10-12$ & $11-12$ \\
\hline Head diam. & $8-9$ & 8 \\
\hline Mouth diam. & 1 & 1 \\
\hline Length of cephalic setae & $3-4$ & $2-3$ \\
\hline Amphid height & 5 & $4-5$ \\
\hline Amphid width & $5-6$ & 5 \\
\hline Amphid width/cbd (\%) & $56-66$ & 63 \\
\hline Amphid from anterior end & $2-3$ & $2-3$ \\
\hline Nerve ring from anterior end & $80-85$ & $79-90$ \\
\hline Nerve ring cbd & $23-25$ & $23-28$ \\
\hline Excretory pore from anterior end & $97-107$ & $94-116$ \\
\hline Pharynx length & $141-150$ & $138-160$ \\
\hline Pharynx cbd & $28-31$ & $29-33$ \\
\hline Pharyngeal bulb diam. & $14-17$ & $16-18$ \\
\hline Max. body diam. & $30-39$ & $39-43$ \\
\hline Spicule length & $45-49$ & - \\
\hline Gubernacular apophyses length & $11-15$ & - \\
\hline Anal body diam. & $27-29$ & $26-28$ \\
\hline Tail length & $90-99$ & 96-105 \\
\hline Tail length/abd & $3.2-3.7$ & $3.5-3.8$ \\
\hline $\mathbf{V}$ & - & $569-644$ \\
\hline$\% \mathrm{~V}$ & - & 53 \\
\hline Vulval body diam. & - & $39-43$ \\
\hline
\end{tabular}

buccal cavity ( 4 vs. $8 \mu \mathrm{m}$ deep) and are characterised by the presence of 2-3 pre-cloacal supplements. No pre-cloacal supplements were observed in the original description of this species, but may have been overlooked. This is the first record for this genus outside the type locality.

\section{Discussion}

A recent study suggests that at least 775 nematode morphospecies (species that can be differentiated from others based on their morphology but which have not been formally described) are present on the continental margins of New Zealand (water depth 250-3100 m; Leduc et al. 2012b). Such high level of diversity is probably related to the wide range of environments encompassed by the country's Exclusive Economic Zone (e.g., Nodder et al. 2012). However, only 12 nematode species (or 1.5\% of total) have been recorded/described from New Zealand's deep-sea habitats so far (Wieser 1956, Leduc et al. 2012a, present study). More work is clearly needed to obtain a better appreciation of New Zealand's deep-sea nematode fauna and its relationship to the fauna of other regions.

New records of $C$. shiae Chen \& Vincx, 2000, H. beaglense Chen \& Vincx, 1998, and K. monorchis Muthumbi et al., 1997 on the continental margin of New Zealand suggest that these species have a 
wide distribution extending across ocean basins. Miljutin et al. (2010) noted that, out of 638 formally described deep-sea nematode species, 46 (including three Comesomatidae species) are likely to have a cosmopolitan distribution. The true proportion of deep-sea cosmopolitan species remains unknown due to paucity of data. Recent molecular evidence provides support for the existence of cosmopolitan nematode species in the deep sea (Bik et al. 2010), although the vast majority of families (including the Comesomatidae) remains to be investigated.

\section{Acknowledgments}

Sample data were generated under the Foundation for Research, Science and Technology (FRST) programme "Ocean Ecosystems" (C01X0027) and the Ocean Survey 20/20 Chatham-Challenger project for which I thank the funding agencies, Land Information New Zealand, Ministry of Fisheries, Department of Conservation and NIWA, for permission to use the data. Funding was also provided by FRST through a postdoctoral fellowship (UOOX0909), the programmes "Consequences of Earth-Ocean Change" (C01X0702), "Coasts \& Oceans OBI" (C01X0501), and "Impact of resource use on vulnerable deep-sea communities" (CO1X0906). I also acknowledge the other participants of voyages TAN0116, TAN0705, TAN0707, TAN1006 and the officers and crew of RV Tangaroa. I thank P. Keith Probert (University of Otago) and Scott Nodder (NIWA) for supporting my research, Katrin Berkenbusch (University of Otago) for processing of meiofauna samples (TAN0116). I am also grateful to the editors and two anonymous reviewers for their helpful comments on the manuscript.

\section{References}

Barnes N., Kim H. G. \& Lee W. 2012. New species of free-living marine Sabatieriinae (Nematoda: Monhysterida: Comesomatidae) from around South Korea. In: Karanovic T. \& Lee W. (eds) Biodiversity of Invertebrates in Korea. Zootaxa 3368: 263-290.

Bik H.M., Thomas W.K., Hunt D.H. \& Lambshead P.J.D. 2010. Low endemism, continued deepshallow interchanges, and evidence for cosmopolitan distributions in free-living marine nematodes (order Enoplida). BMC Evolutionary Ecology 10: 389. http://dx.doi.org/10.1186/1471-2148-10-389

Bradford-Grieve J.M., Chang F.H., Gall M., Pickmere S. \& Richards F. 1997. Size-fractionated phytoplankton standing stocks and primary production during austral winter and spring 1993 in the Subtropical Convergence region near New Zealand. New Zealand Journal of Marine and Freshwater Research 31 (2): 201-224. http://dx.doi.org/10.1080/00288330.1997.9516759

Chen G. \& Vincx M. 1998. Nematodes from the Strait of Magellan and the Beagle Channel (Chile): Description of four new species of the Comesomatidae. Hydrobiologia 379: 97-110. http://dx.doi. org/10.1023/A:1003494127181

Chen G. \& Vincx M. 2000. Nematodes from the Strait of Magellan and the Beagle Channel (Chile): the genera Cervonema and Laimella (Comesomatidae: Nematoda). Hydrobiologia 427 (1): 27-49. http:// dx.doi.org/10.1023/A:1003943021901

De Leo F.C., Smith C.R., Rowden A.A., Bowden D.A. \& Clark M.R. 2010. Submarine canyons: hotspots of benthic biomass and productivity in the deep sea. Proceedings of the Royal Society B: Biological Sciences 277: 2783-2792. http://dx.doi.org/10.1098/rspb.2010.0462

Hope W.D. \& Zhang Z. 1995. New nematodes fom the Yellow sea, Hopperia hexadentata n. sp. and Cervonema deltensis n. sp. (Chromadorida: Comesomatidae), with observations on morphology and systematics. Invertebrate Biology 114: 119-138.

Jensen P. 1979. Revision of Comesomatidae (Nematoda). Zoologica Scripta 8: 81-105. http://dx.doi. org/10.1111/j.1463-6409.1979.tb00621.x 
Leduc D. \& Wharton D.A. 2008. Three new species of free-living nematodes from inter-tidal sediments in southern New Zealand. Nematology 10 (5): 743-755. http://dx.doi.org/10.1163/156854108785787163

Leduc D., Probert P.K. \& Nodder S.D. 2012a Two new free-living nematode species (Comesomatidae) from the continental slope of New Zealand, with keys and notes on distribution. Zootaxa: 3348: 40-55.

Leduc D., Rowden A.A., Bowden D.A., Nodder S.D., Probert P.K., Pilditch C.A., Duineveld G.C.A. \& Witbaard R. 2012b. Nematode beta diversity on the continental slope of New Zealand: spatial patterns and environmental drivers. Marine Ecology Progress Series 454: 37-52. http://dx.doi.org/10.3354/ meps09690

Lorenzen S. 1981 Entwurf eines phylogenetischen Systems der freilebenden Nematoden. Veröffentlichungen des Instituts für Meeresforschung in Bremerhaven Supplement 7: 1-472.

Miljutin D.M., Gunnar G., Miljutina M.M., Mokievsky V., Fonseca-Genevois V. \& Esteves A.M. 2010. The state of knowledge on deep-sea nematode taxonomy: how many valid species are known down there? Marine Biodiversity 40 (3): 143-159. http://dx.doi.org/10.1007/s12526-010-0041-4

Murphy R.J., Pinkerton M.H., Richardson K.M. \& Bradford-Grieve J.M. 2001. Phytoplankton distributions around New Zealand derived from SeaWiFS remote-sensed ocean colour data. New Zealand Journal of Marine and Freshwater Research 35 (2): 343-362. http://dx.doi.org/10.1080/00288 $\underline{330.2001 .9517005}$

Muthumbi A.W., Soetaert K. \& Vincx M. 1997. Deep-sea nematodes from the Indian Ocean: new and known species of the family Comesomatidae. Hydrobiologia 346: 25-57.

Muthumbi A.W., Vanreusel A. \& Vincx M. 2011. Taxon-related diversity patterns from the continental shelf to the slope: a case study on nematodes from the Western Indian Ocean. Marine Ecology 32 (4): 453-467. http://dx.doi.org/10.1111/j.1439-0485.2011.00449.x

Pastor de Ward C.T. 2004. New species of Hopperia (Nematoda, Comesomatidae) and Metachromadora (Nematoda, Desmodoridae) from Patagonia, Chubut, Argentina. Zootaxa 542: 1-15.

Platt H.M. 1984. The freeliving marine nematode genus Sabatieria (Nematoda: Comesomatidae). II. Redescriptions of five European species. Bulletin of the British Museum of Natural History (Zoology) 46: 355-375.

Riemann F. 1977. On the excretory organ in Sabatieria (Nematoda, Chromadorida). Veröffentlichungen des Instituts für Meeresforschung in Bremerhaven 16: 263-267.

Soetaert K. \& Heip C. 1995. Nematode assemblages of deep-sea and shelf break sites in the North Atlantic and Mediterranean Sea. Marine Ecology Progress Series 125: 171-183. http://dx.doi.org/10.3354/ meps 125171

Somerfield P.J. \& Warwick R.M. 1996. Meiofauna in marine pollution monitoring programmes: a laboratory manual. Ministry of Agriculture, Fisheries, and Food, Lowestoft.

Vanhove S., Arntz W. \& Vincx M. 1999. Comparative study of the nematode communities on the southeastern Weddell Sea shelf and slope (Antarctica). Marine Ecology Progress Series 181: 237-256. http://dx.doi.org/10.3354/meps181237

Wieser W. 1954. Free-living marine nematodes. II. Chromadoroidea. Acta Universitatis Lundensis 50, C.W.K. Gleerup, Lund.

Wieser W. 1956. Some free-living marine nematodes. Galathea Report 2: 243-253.

Zhang Z. 1992. Two new species of the genus Dorylaimopsis Ditlevsen, 1918 (Nematoda: Adenophora, Comesomatidae) from the Bohai Sea, China. Chinese Journal of Oceanology and Limnology 10 (1): 31-39. http://dx.doi.org/ 10.1007/BF02844297 
Manuscript received on: 25 June 2012

Manuscript accepted on:4 September 2012

Published on: 5 October 2012

Topic editor: Rudy Jocqué

In compliance with the $I C Z N$, printed versions of all papers are deposited in the libraries of the institutes that are members of the EJT consortium: Muséum national d'Histoire naturelle, Paris, France; National Botanic Garden of Belgium, Meise, Belgium; Royal Museum for Central Africa, Tervuren, Belgium; Natural History Museum, London, United Kingdom; Royal Belgian Institute of Natural Sciences, Brussels, Belgium; Natural History Museum of Denmark, Copenhagen, Denmark. 